\title{
Chapitre 10 En arrière plan de la RDA, le « discours autre » constitutif du dire : deux pensées de l'extériorité interne au dire
}

Dans l'ensemble des approches ouvrant le dire sur ses extériorités internes, deux courants s'imposent : le dialogisme et l'AD, en ce que, derrière des objets aussi différents que les genres littéraires pour Bakhtine et les processus idéologiques pour Pêcheux, ce sont, d'abord, des théorisations du langage et du sens qui sont élaborées : pour l'un comme pour l'autre, la parole, dans sa linéarité formelle, ne peut pas se penser hors de la matérialité discursive historique, condition du dire et du sens, dans laquelle elle se constitue. Par là, ils constituent l'un et l'autre des références obligées pour la prise en compte solidaire des deux niveaux, de la chaîne - observable dans son déroulement linéaire - et de l'extériorité discursive - envisagée comme espace, milieu, corps...

Mais si la reconnaissance de ces deux plans est partagée, il n'en va pas de même pour les modalités sous lesquelles de part et d'autre est pensée leur mise en rapport : en termes de " rencontre » et « dialogue » du dire avec l'extérieur discursif, d'un côté, là où de l'autre elle relève de la « détermination » du dire par cet extérieur.

La complexité des différences de problématiques, objets, méthodes, qui passent à travers la pluralité des auteurs et des " moments » d'évolution de ces deux courants, qui demande de préciser le mode sur lequel on peut tenter de les " articuler », incite en même temps à une prudente modestie... Je ne vise pas ici à rendre compte de façon globale, même schématique, de chacun d'eux, mais seulement à faire apparaître, à travers la présentation de l'un puis de l'autre (1 et 2) quelques-uns des points essentiels d'appui que - dans l'immense " déjà-dit » des élaborations du « non-un » du dire - la formulation du couple représenté/ constitutif a pu trouver en eux (plutôt que dans d'autres approches interactionnelles ou polyphoniques), mais aussi des limites et des difficultés rencontrées à cet appui, différemment, dans l'un et l'autre de ces courants ou dans un moment plutôt qu'un autre de leur trajectoire... ${ }^{1}$, notamment touchant la question du sujet (3) crucialement en jeu dans ce rapport du dire à son « ailleurs » langagier.

1 Dans la même visée spécifique d'ancrage de l'opposition représenté/constitutif, on trouve des présentations plus étoffées de ces deux courants dans Authier-Revuz (1982), (1995/2012).

2 Open Access. (C) 2020 Jacqueline Authier-Revuz, published by De Gruyter. (cc)BY-NC-ND This work is licensed under the Creative Commons Attribution-NonCommercial-NoDerivatives 4.0 License. https://doi.org/10.1515/9783110641226-019 


\section{1 «L'autre dans l'un » de la traversée dialogique du déjà-dit}

Une unité se dégage de la pensée multiforme du « dialogisme $»^{2}$ : problématiques philosophiques, éthiques, épistémologiques, littéraires, langagières sont de part en part portées par une pensée du rejet du UN, comme antinomique du pluriel, de l'hétérogène, du relatif, du conflictuel, de l'inachevé, posés comme le fait même $\mathrm{du}$ « vivant ».

$\mathrm{Si}$, dans le champ langagier, cette pensée de " l'autre dans l'un » trouve ses objets de prédilection dans les formes de langue du discours rapporté et les genres textuels « polyphoniques », ceux-ci ne sont jamais « coupés » du réel dans lequel ils s'ancrent et dont ils apparaissent comme des « révélateurs », ou des images : le « dialogisme » foncier du langage, articulant (1.1) le travail différenciateur - hétérogénéisant - de l'histoire dans la langue, à (1.2) la dynamique des « rencontres » du dire, dans son mouvement à travers le déjà-dit.

\subsection{Le « milieu du déjà-dit », produit du travail de l'histoire}

Le travail de l'histoire - «la vie sociale vivace et le devenir historique ${ }^{3}$ - dans le UN de la langue, celui qu'opèrent les discours, est d'abord celui de la «stratification », processus de différenciation à « l'intérieur d'une langue nationale

2 Dans la masse des travaux autour de ceux - Bakhtine, Voloshinov, Medvedev - qu'on a rassemblé comme représentants du « dialogisme » je me contente de rappeler Kristeva (1970), Todorov (1981), Peytard (1995) qui en ont marqué la réception en France, et de souligner le mouvement récent de réévaluation critique " recontextualisante » - tant historique qu'épistémologique - de ce courant et de sa réception (entre autres de la consistance de la notion de « cercle Bakhtine »), notamment autour de P. Sériot : cf. Sériot (2007), Sériot et al. (2008), Sériot (préface à Voloshinov (2010) ; cf. aussi Bouquet et al. (2007), Bronckart et al. (2011). Je considère d'emblée comme écartée la confusion entre « dialogal »-forme extérieure de l'alternance des voix dans l'échange interlocutif - et dialogisme - dimension inhérente au fait langagier, quelle que soit la forme sous laquelle il se réalise, $c f$. par exemple, les articles « Dialogisme » et « Dialogue » in DAD 2002 ou Bres et al. (2005: 36-38). L'aspect qui me retient ici - celui de l'ancrage « dialogique » du dire dans le milieu historiquement constitué du déjà-dit - m’apparaît, au niveau de généralité où je me situe, suffisamment partagé par l'ensemble de ce courant - réparti, entre trois auteurs d'abord, puis sur 40 ans de production de l'un d'eux - pour que je fasse abstraction des différences réelles qui peuvent s'y observer, et suffisamment " massif " pour que la lecture que j'en propose ici, prolongeant et s'appuyant sur celles de Authier-Revuz (1982: 101-123) et (1995/2012: 249-254), plus détaillées, n'encoure pas trop le risque d'une vraie remise en cause par les éclairages récents évoqués ci-dessus. 3 Bakhtine (1975, Du discours romanesque : 110), texte sur lequel je m’appuie centralement. 
abstraitement unique ». Le pluri/multilinguisme inhérent à toute langue ${ }^{4}$, si volontiers parcouru par Bakhtine dans des inventaires de parlers « socialement typiques $»^{5}$, ne se ramène pas à celui d'une variété dialectale perçue comme « habillage » diversifié pour des contenus semblables : il fait de la langue « une opinion multilingue sur le monde $»^{6}$ : car ce sont aussi des «perspectives » ou des « points de vue spécifique sur le monde, des formes de son interprétation verbale ${ }^{7}$ que portent en eux les « langages du plurilinguisme ». Les « langages sociaux » ne coexistent pas au sein d'une même langue sur le mode d'une marqueterie stabilisée mais dans la constante instabilité de rapports " d'imbrication ", " d'intersection ", " d'entrecroisements », formant des « parlers neufs, socialement typiques $»^{8}$.

Ce travail est ensuite celui de la mémoire dans laquelle se produit ce que Bakhtine appelle la saturation du langage : un espace dans lequel aucun mot n'échappe à l'emprise du « déjà-dit » dont il est « habité », « environné », « saturé »... :

Comme résultat du travail de toutes ces forces stratificatrices, le langage ne conserve plus de formes ou de mots neutres « n'appartenant à personne ». [...] Chaque mot renvoie à son contexte ou à plusieurs dans lesquels il a vécu son existence socialement sous-tendue? ${ }^{9}$.

de la même façon qu'aucun « objet » ne se présente " neutre », c'est-à-dire « innommé », mais

comme déjà spécifié, contesté, évalué, emmitouflé [...] par les paroles étrangères à son propos. Il est entortillé, pénétré par les idées générales, les vues, les appréciations, les définitions d'autrui ${ }^{10}$.

4 « [... qui] doit se stratifier intérieurement à tout moment de son existence historique » (ibid. : 88-89).

5 (ibid. : 112), $c f$. aussi : « La langue nationale se stratifie en dialectes sociaux, en manières d'un groupe, en jargons professionnels, langages des genres, parlers des générations, des écoles, des autorités, cercles et modes passagères, en langage des journées (voire des heures) sociales, politiques (chaque heure possède sa devise, son vocabulaire, ses accents [...]. » (ibid. : 88)

6 (ibid. : 114).

7 (ibid. : 113).

8 (ibid. : 99).

9 (ibid.: 114), cf. l'image chez Valéry de mots « desquels la mémoire est barbouillée » de ce qu'ils ont « fait tous les métiers » (Regards sur le monde actuel - Fluctuations sur la liberté, 1931)).

10 (ibid. : 100). C’est, par exemple, ce qu'évoque - « fond » rhétorique propre à faire se détacher une singularité narcissique affirmée - cette ouverture d'article de B.H. Lévy consacré à l'évocation d'un séjour à Rome : " Rome et les ruines... Rome et sa mémoire... Rome et son histoire... Rome la morte, la mortelle, la morbide... "La base de Rome est un tombeau” disait Michelet... [...] C’est tout ça que j'avais en tête au début de cette dérive romaine. Tous ces textes. Tous ces sou- 
Ainsi la sédimentation de déjà-dit qu'opère la mémoire de l'usage des mots, en contexte, façonne-t-elle - en y inscrivant de la diversité et du conflit - tout à la fois la polysémie ${ }^{11}$ de mots " pluri-accentués ", " arènes en réduction où s'entrecroisent et luttent les accents sociaux à orientation contradictoire ${ }^{12}$, et la pluralité des nominations renvoyant à un même objet, porteuses de leur appartenance discursive et, avec elle, de « points de vue » diversifiés sur le monde.

\subsection{La dynamique du dire : rencontres dialogiques dans la traversée " orientée " du déjà-dit}

« Orienté sur son objet », un énoncé va prendre corps dans - et de - son trajet de traversée de ce milieu du déjà-dit : telle est la loi du discours humain - à laquelle seul peut échapper « l'Adam mythique abordant avec le premier discours un monde vierge et encore non dit $»^{13}$.

$\mathrm{Au}$ cœur du surgissement du dire, s'impose la « résistance » du déjà-dit : cette résistance se joue, pour Bakhtine, sur le mode de l'interaction obligée - « rencontre » et réaction - avec le discours d'autrui :

Le discours rencontre le discours d'autrui sur tous les chemins qui mènent à son objet, et il ne peut pas ne pas entrer avec lui en interaction vive et intense ${ }^{14}$.

Rencontres avec les mots, expressions, accents, jugements " étrangers » qui tissent le déjà-dit et réactions d'accord, désaccord, évitement etc. du discours qui se « faufile » dans leurs interactions compliquées, fusionne avec les uns, se détache des autres, se croise avec les troisièmes, forment le « processus de dialogisation » dans lequel le discours « peut s'individualiser et s'élaborer stylistiquement », trouver sa « forme » et son « ton », c'est-à-dire « se faire » - au sens plein du terme.

On notera que c'est encore en termes de rapports entre discours constitués dans le déjà-dit, qu'est pensé le rapport interlocutif : loin du modèle télégraphique de transmission au récepteur d'un message tout fait, étranger aussi aux stratégies

venirs. [...] dans ce cas-ci on évite difficilement le lieu commun [B.H. Lévy, Vacances Romaines, Maison de Marie Claire, mai 1985]».

11 [...] d'identiques éléments abstraits du langage se chargent de différents contenus sémantiques et axiologiques et résonnent différemment. (ibid. : 110)

12 Voloshinov (1929: 67).

13 Bakhtine (1975), traduction du fragment dans Todorov (1981 : 98).

14 ibid. 
communicationnelles en termes de contenus, la visée du destinataire intégrée à la production du discours se situe chez Bakhtine au plan de sa « compréhension répondante », c'est-à-dire du discours-réponse prêté à l'autre ; les deux relations au déjà -dit, qu'elles soient " aimantées » par l'objet visé ou par l'interlocuteur « peuvent, note Bakhtine (1975 : 105), s'entrelacer très étroitement » : le dialogisme « interlocutif » apparaît comme partie prenante d'un dialogisme complexe où il intervient comme facteur - discursif - spécifique d'orientation du cheminement dialogique d'ensemble du discours dans le déjà-dit ${ }^{15}$.

Réfracté dans la « conscience individuelle », le processus de constitution du discours dans le déjà-dit apparaît - loin de la tranquille « utilisation » par le sujet parlant de mots mis à sa disposition par la langue - comme l'expérience d'une appropriation, ni aisée, ni achevée, laissant au cœur du dire l'écart d'une dépossession :

[...] pour la conscience individuelle [...] le mot du langage est un mot semi-étranger. [...] Jusqu'au moment, où il est approprié [...] il est sur des lèvres étrangères, dans des contextes étrangers, au service d'intentions étrangères, et c'est là qu'il faut le prendre et le faire "sien". [...] Le langage [...] n'est pas un milieu neutre. Il ne devient pas aisément, librement, la propriété du locuteur ${ }^{16}$.

et,

L'auteur (le locuteur) a ses droits inaliénables sur le discours, mais [...] en ont aussi ceux dont les voix résonnent dans les mots trouvés par l'auteur (puisqu'il n'existe pas de mots qui ne soient à personne) [...] Le discours [...] se joue en dehors de l'auteur, $[. . .]^{17}$.

15 Cf. Authier-Revuz (1995/2012) ; Bres (1998 : 44) note justement que dans l'approche bakhtinienne des deux dialogismes «l'interdiscursif assimile quelque peu l'interpersonnel : la première dialogisation tient à la rencontre des discours d'autrui, la seconde à la rencontre d'autrui comme discours ». De fait, cette « discursivisation » intégrale du rapport intersubjectif apparait en particulier réductrice relativement à ce qui se joue - « l'impossible conjonction de sujets désirants (Milner 1978) - de non réductible à la discursivité, entre des sujets marqués par l'Autre de l'inconscient, $c f$. Authier-Revuz (1982 : 119) et (1995/2012 : 176-178).

16 Bakhtine (1975 : 114-115). Cf. aussi : « Il [le langage] est peuplé et surpeuplé d’intentions étrangères. Le dominer, les soumettre à ses intentions et accents, c'est un processus ardu et complexe » (ibid.).

17 Bakhtine (1979 : 331), trad. Todorov (1981 : 83). 


\subsection{Le dialogisme : une cohérence à ne pas tronquer}

Le dialogisme bakhtinien repose donc sur cette conception d'une matérialité historique de la discursivité qui, déposée dans une langue "plurilinguisée », est constitutive, en extériorité interne, de tout fait de discours qui y trouve sa forme et son sens : une des séductions et des forces de ce courant réside dans l'étendue du spectre sur lequel se déploie, à des plans divers - linguistique ou textuel - cette inscription du jeu de «l'autre dans l'un » au principe du fait langagier.

Ainsi, les formes du discours rapporté, envisagées comme fonctionnements dialogiques " grammaticalisés » dans la langue, apparaissent-elles, en aboutissement de l'ouvrage de Voloshinov, comme manifestation privilégiée du fonctionnement "socio-interactif » inhérent au langage. De même, si au plan textuel le roman est la forme littéraire privilégiée par Bakhtine, c'est en tant que sa vocation est, à ses yeux, de représenter le langage, de produire une image stylisée de son fonctionnement dialogique ${ }^{18}$ : le roman " polyphonique » orchestré par Dostoïevski, et au-delà l'horizon idéal du roman «tout entier entre guillemets » apparaissent comme des accomplissements du genre, au plus près du dialogisme foncier du langage.

La cohérence et la transversalité de la pensée de Bakhtine, saisissant d'un même mouvement les divers plans du langage, apparaît encore de façon frappante à propos de la notion, dialogique par excellence, de « l'hybridisation » comme mélange, amalgame de «deux langages » ou accents, voix, parlers ${ }^{19}$ : si son « étage » d'hybride « intentionnel », conscient, est spécifié par la mise en jeu d'une « conscience représentante » à l'œuvre dans la création d'une « image dialogisée du langage » - dont il apparaît comme un des principaux « procédés ${ }^{20}-$, c'est dans un constant va et vient que, " [hybride] concret et social », il est ancré dans le fait de l'hybridation « historico-organique », involontaire, " obscure », loin de la « juxtaposition » et de l'opposition consciente entre deux langages qui

18 «[...] le problème central de la stylistique du roman peut être formulé comme problème de la représentation littéraire du langage, problème de l'image du langage. (Bakhtine 1975 : 156, idt)». " L'objet principal du genre romanesque, ce qui le spécifie et qui crée son originalité stylistique, c'est l'homme qui parle et sa parole » (Bakhtine 1975 : 153, idt).

19 Bakhtine (1975 : 175-178).

20 Ces mélanges de voix que permet une forme - tels, par exemple, ceux que structurent le mode Bivocal et la MAE - approchés avec acuité par Bakhtine, ne se situent pas du tout au même plan que les hybrides de formes (i.e. mélanges de DD/DI, par exemple) proposés parfois pour rendre compte de certaines configurations complexes (rappelons, $c f$. ci-dessus, chap. 4.3.1, p. 132, la position de Voloshinov sur le DIL comme forme autonome et non pas « mixte » de DD et de DI). 
est, avec ses « mélanges épais et sombres », l'un des « modes majeurs » de « l'existence historique » et du devenir « stratifié » des langues « multilingues ${ }^{21}$.

Là où la solidarité de l'approche de «l'autre en discours » comme loi et condition d'existence et comme objet - linguistique ou textuel - de représentation, s'impose, à mon sens, comme une des spécificités stimulantes du dialogisme bakhtinien, c'est pourtant à une lecture partielle, dissociante, négligeant - ou rejetant - le premier versant, que relèvent bien des emplois de ce terme - trop ? parlant. Une "vulgate » du dialogisme (ou tout au moins des usages du terme « dialogique ») le restreint en effet à ce que l'on pourrait appeler les manifestations « horizontales » - sur la chaîne - de l'autre représenté, à l'exclusion de l'épaisseur de déjà-dit qui « verticalement » la travaille : de la sphère du « discours dans le discours » du dialogisme, n'est alors retenue que la part qui en est " passée » par le filtre de la conscience métalangagière - du discours sur du discours. Il en va ainsi de définitions du dialogisme comme propriété de certains énoncés, ou de l'opposition, couramment formulée, entre énoncés (ou textes) « dialogiques vs monologiques $»^{22}$, dont, dans un cadre strictement bakhtinien, le premier terme est tautologique et le deuxième contradictoire ${ }^{23}$, puisque, selon la formulation chère à Bakhtine, un énoncé « ne peut pas ne pas » être dialogique...

Ces formulations ne peuvent, dans le cadre bakhtinien, renvoyer à autre chose qu'à l'image de dialogisme - omniprésent/nourri/faible/absent... - que les discours présentent en eux-mêmes ; c'est ainsi qu'en face du genre romanesque, s'accomplissant pour Bakhtine, on l'a vu, dans une représentation maximale du dialogisme, la voix poétique qui, à l'opposé selon lui, trouve son achèvement dans l'unité, l'identité, « d'une pleine solitude », n'y accèderait que par une tension stylistique de création de « un » par « enlèvement », mise en silence, des voix d'autrui inévitablement rencontrées :

\section{Bakhtine (1975: 176, 177, idt).}

$22 C f$., par exemple : « Le terme de dialogisme (idt) est employé dans la théorie des textes, pour caractériser la propriété qu'ont certains énoncés de n'être pas attachés à une seule mais à diverses sources d'énonciation. Les travaux du formaliste russe Mikhail Bakhtine [...] ont montré que l'occurrence ponctuelle d'une ou plusieurs phrases pouvait être déterminée non seulement par l'acte de parole [...] d'un locuteur [...] singulier, mais aussi par des actes antérieurs émanant d'instances plus ou moins identifiées » (Jeandillou 1997 : 76). «[...] dans un énoncé monologique le locuteur est aussi énonciateur ; dans un énoncé dialogique, peuvent coexister un certain nombre de locuteurs/ énonciateurs seconds [...] (Rabatel 2008b : 8), où l'auteur - dans ce texte - situe cette opposition au plan, inspiré de Ducrot, de la «mise en scène énonciative ». Voir, au contraire, la mise au point de S. Moirand à l'article « Dialogisme » de (DAD 2002 : 76).

23 Ce que rejoint la formulation heureuse de J. Bres (2005a) : « le monologique tend à être une catégorie vide ». 
[...] ce cheminement du discours poétique vers son objet et vers l'unité du langage, cheminement au cours duquel il rencontre lui aussi, continuellement, le discours d'autrui et s'oriente mutuellement avec lui, demeure dans les scories du processus de création, et s'enlève comme s'enlèvent les échafaudages d'un bâtiment terminé ; alors l'œuvre parachevée s'élève, tel un discours unique et concentré sur un objet, un discours, sur un monde « vierge $»^{24}$.

Ainsi, parler de « dialogisme », de " discours dialogique/monologique » en référence aux seules couches qui en donnent une représentation relève-t-il, relativement à la pensée bakhtinienne du dialogisme, de la commodité d'un abus de langage (préférant le bref « discours monologique » à « discours qui se représente comme/se donne pour monologique »), avec les risques de confusion entre les deux plans que cela comporte ; au-delà les références au dialogisme ou à Bakhtine dans des approches stylistiques ou, plus encore, dans les traitements pragmatiques et/ou conversationnels de l'altérité énonciative qui posent - de fait, ou explicitement - la question du rapport au dire de l'autre au niveau intralinguistique ou interlocutif, à l'exclusion de toute prise en compte de l'extériorité discursive, apparaissent, tournant le dos à ce qui constitue l'ancrage même de la pensée bakhtinienne, comme non pertinentes, et l'amputation déshistoricisante qu'elles pratiquent sur celle-ci comme un avatar. de la « très ancienne élision de la réalité du discours » évoquée par Foucault. ${ }^{25}$

Remarque 1 : Polyphonie de Ducrot et déjà-dit bakhtinien. C'est dans cette élision de l'extériorité discursive interne au dire que, foncièrement, se fait l'écart - formulé en termes « d'extension (très libre) » par Ducrot (1984: 173) - entre approche polyphonique de l'énonciation et dialogisme bakhtinien.

24 Bakhtine (1975 : 150). Cette dernière phrase fait jouer, à l'horizon de la pratique poétique, l'image du " solitaire Adam », etc..., mais ramenée au réel du langage humain par le tel et le guillemet sur vierge. On peut, certainement, contester cette conception bakhtinienne du poétique, cf. Authier-Revuz (1995/2012 : 663) : elle n'est, ici, convoquée que pour éclairer le rapport posé par Bakhtine entre le fait - obligé - de la traversée du déjà-dit et l'image « une » qu'en offrirait, selon lui, ce type de discours.

25 Et on ne doit pas s'étonner que les « défenseurs » de « l'épaisseur » historique du dialogisme soient aussi, parmi les héritiers de Bakhtine, ceux qui se réclament aussi, diversement, de l'AD et/ ou de la praxématique : ainsi, P. Schepens (2006b : 38) qui souligne « la dimension diachronique et transformationnelle [au sens d'une rencontre transformatrice, pas au sens génératif bien sûr] qu'il donne à toute prise de parole » dans ce qui fait la « force » de la pensée de Bakhtine, ou J. Bres dont la résistance vigoureuse à la « dissolution » du dialogisme «dans les eaux accueillantes du calcul communicatif » traverse les textes récents : Bres (2004, 2006), Bres et Nowakoska (2005, 2008). Cf. Remarque ci-dessous. 
Si le caractère tranché du choix explicité par Ducrot de situer son approche « polyphonique » de l'énonciation au plan intralinguistique d'énoncés-phrases détachés de tout ancrage subjectif et historique ${ }^{26}$ s'oppose évidemment à la perspective de l'AD, comme le rappelle Pêcheux (1990 : 291) :

O. Ducrot se refuse absolument à faire intervenir dans l'analyse linguistique de la séquence la référence à quelque corpus interdiscursif que ce soit : il fait même de ce refus un critère distinctif de ce qui peut se présenter légitimement comme une analyse linguistique.

il apporte, en même temps, la plus nette des restrictions à une véritable «filiation » bakhtinienne de Ducrot : par delà l'emprunt en forme d'hommage du mot « polyphonie », il faut reconnaître ce qui sépare - voire oppose - pluralité des voix orchestrée dans une mise en scène en quoi consiste une énonciation chez l'un, et, chez l'autre, dire partagé en tant qu'il prend forme dans et de son inévitable traversée d'un « milieu » de déjà-dit, historiquement constitué...

On ne saurait, aussi, suivre Ducrot lorsque - ne retenant du large « spectre » du dialogisme que la stylisation qu'en proposent certaines œuvres caractérisées comme « polyphoniques » - il réduit l'observation du fait - dialogique - de la pluralité des voix à l'œuvre dans un dire par Bakhtine, au seul plan des textes,

jamais aux énoncés dont ces textes sont constitués. De sorte qu'elle n’a pas abouti à mettre en doute le postulat selon lequel un énoncé isolé fait entendre une seule voix. (1984 : 171).

Non seulement la notion « d'hybridisation » évoquée ci-dessus, touche à l'évidence les énoncés, mais au-delà, on l'a vu, c'est en tout mot énoncé que résonnent « inévitablement » les voix, déposées en lui, du déjà-dit, qui « altèrent » le dire du « sujet parlant ».

Je renvoie au parcours, dans Bres et Rosier (2007) de la « réfraction » des concepts bakhtiniens, dans le cadre « des reconfigurations théoriques » ayant accompagné leur réception dans l'espace francophone, notamment (p. 445-448) à la partie « Ducrot et la notion de polyphonie : le regard éloigné $»^{27}$.

En ce qu'elle est « délestée » du souci bakhtinien du milieu du déjà-dit où se forme le dire, la polyphonie de Ducrot apparaît étrangère à ce « socle » que la notion d'hétérogénéité constitutive peut trouver, en deçà de leurs divergences, chez Bakhtine et Pêcheux. On revient plus loin - $c f$. 3.4.2 ci-dessous - sur les autres choix, au plan du sujet, de l'énonciation, du sens, qui sous d'apparentes similitudes de formulation (une « altérité interne » du dire ayant « valeur constitutive » (Ducrot,1984) confortent l'incompatibilité théorique entre approche polyphonique et articulation représenté/constitutif.

26 Cf. Ducrot (1980, 1984), clairement formulé dans sa discussion avec P. Henry (Henry (1977), notamment p. 202-203)

27 Prenant la mesure de la « liberté » que reconnaît Ducrot dans « l'extension » opérée par la polyphonie à partir de Bakhtine, ils opposent (p.448) à la « pluralité des voix permettant dans le cadre structuraliste de l'énoncé-phrase de rester à l'intérieur du champ de la linguistique pragmatique ", mise en œuvre chez lui, à ce qui, ailleurs, relève d'un « rapport incontournable aux autres discours et aux sens déjà produits dans les mots ", concluant que les deux façons dont le non-un est traité « n’ont pas grand chose à voir ». Cf. aussi la précise mise au point de Sitri (2004). 


\subsection{Questionnements descriptifs et théoriques}

Envisagé - non tronqué - dans la cohérence de sa problématique globale de « l'autre dans l'un » pour le dire, à la fois aux plans de la loi qui le constitue, et des formes où il se réalise, le dialogisme ouvre des perspectives qui apparaissent en manque des investigations descriptives ou des interrogations théoriques explicites qu'elles appellent.

\subsubsection{La « traversée » constitutive du déjà-dit : postulée mais non décrite}

Dans le processus par lequel le dire prend « forme » dans ce déjà-dit qu'il ne peut pas ne pas traverser, les faits précieusement analysés chez Voloshinov et Bakhtine - discours rapporté, guillemets, formes littéraires « bivocales » du pastiche, de la parodie, de la distanciation ironique... - appartiennent, dans l'énonciation, au plan métalinguistique de l'intention « représentante ». C'est un aspect aigu de la pensée de Bakhtine que le lien établi entre position métalinguistique et altérité langagière : lien posé tant au plan théorique dans sa conception d'une « conscience galiléenne » - non à même d'occuper une position « ptoléméenne » extérieure au langage - dans laquelle « le métalangage se rapporte toujours dialogiquement au langage qu'il décrit et analyse ${ }^{28}$, qu'à celui de l'analyse des formes énonciatives, telles celle de «l'hybridation intentionnelle », rendue possible par

cette aptitude d'un langage qui en représente un autre de résonner simultanément hors de lui et en lui, de parler de lui tout en parlant comme lui et avec lui, et d'autre part [à] l'aptitude du langage représenté à servir simultanément d'objet de représentation et de parler par lui-même. (Bakhtine, 1975 : 175).

On l'a souligné plus haut, les formes de la représentation ne sont jamais, dans la cohérence qui fait la force de cette pensée, détachées du fonctionnement dialogique constitutif dont elles sont l'image, ou, littéralement, la stylisation.

Mais la disparité dans l'approche des deux plans - représenté/constitutif est frappante : à la « constitution » dialogique du dire, fortement affirmée ${ }^{29}$ ne répond pas le volet descriptif des fonctionnements discursifs par lesquels elle s’opère : sous quelles formes concrètes se réalisent, dans l'épaisseur du déjà-dit, les processus de « rencontres » diverses avec les mots et les sens des autres, et,

28 Bakhtine, in Todorov (1981: 40).

29 Et qui trouve un écho au plan subjectif dans le « ressenti » au cœur du dire de l'écart subjectif d'une dépossession, $c f$. chap. 13.2. 
en deçà, ceux de la sédimentation multilingue, ou des «mélanges obscurs » de «l'hybridation historico-organique» ? À ces mécanismes suggestivement évoqués ne répond, dans l'approche dialogique, aucune dimension opérative.

C'est du côté des analyses de discours qu'il faut se tourner pour trouver la consistance descriptive qui leur fait défaut dans le corpus bakhtinien ${ }^{30}$; dès lors, en effet, qu'elles font place à la dimension historique de la discursivité, la mise au jour des processus jouant dans « l'épaisseur » hétérogène du déjà-dit est leur domaine par excellence. Que la référence au courant dialogique y soit absente (travaux antérieurs ou élaborés dans un cadre théorique tout autre, comme l'AD) ou présente (inégalement explicitée et combinée selon des pondérations diverses à diverses approches, $\mathrm{AD}$ notamment) ces travaux, de fait, occupent - parcourent, balisent... - cet espace désigné, mais laissé « vacant » par le dialogisme.

Il n'est pas question de rendre justice, même allusivement, à la masse des travaux ayant contribué à donner corps - « sur pièces ", c'est-à-dire en articulant des discours singuliers à (un corpus de) leur mémoire - à la constitution du dire dans le déjà-dit, dont le recensement tendrait à se confondre avec une histoire de l'analyse de discours... Hormis l'AD, envisagée ci-dessous (2), dans sa spécificité, les références qui suivent (Remarque) ne prétendent, sans visée systématique, qu'à marquer par des exemples la permanence et la diversité de cette dimension.

Remarque 2 : Le dialogisme constitutif appréhendé par... les analyses de discours ${ }^{31}$. C'est bien antérieurement à la réception du dialogisme dans les années 80 que la « pluri-accentuation » des mots, " arènes ", divisés par les contextes où ils ont " vécu leur vie de mots ", "s'incarne » dans des analyses de corpus historiquement situé ${ }^{32}$. Ce que B. Gardin (1974: 70) formule en termes de« bataille pour les signes », un mot contre un autre pour nommer le réel, et, pour un mot, un sens contre un autre, est au cœur, dès 1962, de l'étude de J. Dubois, faisant apparaître, en corpus, les clivages de mots et de sens de part et d'autre de la fracture de la Commune ${ }^{33}$, comme

30 Ainsi est-ce le mouvement qu'explicite S. Moirand (2006: 41), en se réclamant de Bakhtine, à l'orée de l'étude empirique d'un corpus de presse : « [...] si le concept tel qu'on peut le dégager des écrits du cercle de Bakhtine est tout à fait fascinant pour construire une réflexion sur le langage et le discours (en philosophie, en littérature, en rhétorique, etc.), Bakhtine ne fournit pas au linguiste de corpus (si ce n'est les formes classiques du discours rapporté) de catégories descriptives qui lui permettent de mettre au jour ses différentes formes d'actualisation dans les données qu'on analyse. »; $c f$. aussi Moirand (1999).

31 Je n'évoque pas ici les travaux spécifiquement inscrits dans le cadre de l'AD (cf. 2. ci-dessous). 32 Maingueneau (1976: 1-98) offre une précieuse présentation des travaux d'analyse de discours publiés à cette date (incluant, p. 93-98, la première mouture de l'approche de Pêcheux).

33 C'est-à-dire comment le lexique « varie suivant les classes, les catégories économiques, les individus et la génération à laquelle ceux-ci appartiennent » et comment « lorsque les classes sociales se servent des mêmes mots elles leur donnent des contenus souvent différents. » (1962:1-2) 
des travaux du Colloque de lexicologie politique de Saint-Cloud en avril $1968^{34}$, mettant au jour, par exemple, les lignes de front traversant le vocabulaire de la guerre d'Algérie, du Congrès de Tours, ou, au siècle précédent, les oppositions pour imposer le « bon » sens du mot « nature » - celui de Rousseau ou de Bonald $-{ }^{35}$; au cœur aussi des analyses du fonctionnement, par exemple, des mots « liberté » par G. Petiot (1988, 1990), ou « grève » par M. Tournier (1982) - c'est-à-dire du mode sur lequel leur sens se divise jusqu'à bloquer « l'échange » verbal entre tenants et ennemis de « l'école libre » pour le premier, ou entre adversaires de classe - euphémiquement nommés «partenaires sociaux » - pour le second.

L'entreprise lexico- ou texto-métrique, appliquant des outils informatiques à des corpus étendus, apparaît dès l'origine ${ }^{36}$ comme une méthodologie de mise en évidence du travail du dire par le déjà-dit : « graphes de co-occurrences » et « segments répétés » donnent corps, préci-

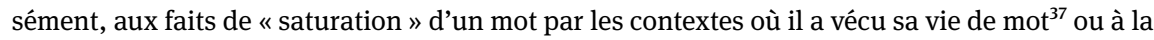
prégnance des formulations déjà-dites offertes « à la redite $»^{38}$.

C'est encore à des aspects du « comment » de la loi de « l'interaction vive et intense » que donnent forme - conceptuelle et concrètement descriptive - des catégories comme « circulation », cristallisation, " formule », dont l'esquisse chez Fiala-Habert (1987) ${ }^{39}$ : « À certaines époques, le débat politique fait circuler des formules qui cristallisent un enjeu fondamental » (p. 45) sera richement approfondie par S. Moirand $(2001,2004)$ et par A. Krieg $(2004,2006)$.

Et c'est explicitement dans la perspective de donner corps, descriptivement, au concept de dialogisme que J. Bres, soulignant que

L'AD doit, plus qu'elle ne l'a fait jusqu'à présent, accorder toute son importance au concept de dialogisme et s'attacher à la description de ses marques (Bres 1999: 83),

inscrit son travail de mise au point dans la linéarité d'une variété de «marqueurs dialogiques ${ }^{40}$, formes de langue se prêtant particulièrement à l'accueil de la mémoire discursive.

34 Publié en 1968-69 dans les Cahiers de Lexicologie.

35 Respectivement dans les communications de D. Maldidier, J.B. Marcellesi, J. Gritti.

36 Notamment au fil de la revue MOTS (Mots, Ordinateurs, Textes, Sociétés), fondée par M. Tournier en 1980, et, par exemple, Salem (1987, 1993), Fiala et al. (1987), Habert et al. (1997).

37 Cf. par exemple, à propos du mot « travail » : « [...] les empilements de texte que la méthode effectue ne donneraient-ils pas une image des moirages de langue dont notre mémoire est le théâtre ? Ce qui tourne dans les graphes c'est un « mémoirage" syndical » (Tournier 1987 : 122).

38 Pour ce très riche ensemble de travaux consacrés à la couche de prêt à dire/écrire - phraséologie, figements, formules, patrons, motifs, structures pré-formées, stéréotypes, clichés... - $c f$. par exemple les tours d'horizon de Amossy et al. (1997), Krieg (2012 : 97-117), Sitri (2015 : 70 sq., 149 sq.). On revient ci-dessous chap. $\mathbf{1 1 . 3}$ sur les rapports entre la RDA et cette " part routinière du langage » (Sitri).

39 « Dans un certain état des rapports de force sociaux, des formules surgissent [...] par rapport auxquelles [...] l'ensemble des locuteurs sont contraints de prendre position, [...] de les combattre ou de les approuver, mais en tout état de cause de les faire circuler.» (Ebel et Fiala, 1983 : 174)

40 Cf. Bres (1998, 1999, 2002, 2005). Envisagées dans une perspective bakhtinienne, ces formes présentent une large intersection avec celles - pensées dans des cadres théoriques différents - 
De la même façon, l'articulation des deux « altérations » constitutives du dire - comme trajet dans le déjà-dit, adressé à un autre ${ }^{41}$ - est un de ces lieux où la mise au travail du principe d'un « double dialogisme » ouvre sur la saisie des formes concrètes sous lesquelles elle se réalise. On peut penser que, retardée par les développements disjoints aussi bien d'une AD longtemps peu soucieuse d'interlocution et que de travaux conversationnels envisageant l'interaction « hors déjà-dit ${ }^{42}$, l'exploration de ce domaine va se développer ${ }^{43}$. Notons qu'un parcours des divers modes de la RDA croise, inévitablement ${ }^{44}$, la dimension du dire adressé, donnant corps, de façon ponctuelle, à cette articulation.

\subsubsection{Quel sujet pour la constitution dialogique du dire dans le déjà-dit ?}

Le déficit descriptif qu'on vient d'évoquer, touchant les processus par lesquels un dire prend corps dans le milieu du déjà-dit, n'est pas seulement à mettre au compte du privilège accordé aux formes de la représentation, notamment littéraires, du dialogisme inhérent au langage ; il apparaît aussi solidaire d'un défaut d'articulation théorique quant au mode sur lequel la subjectivité se trouve inscrite dans la radicalité reconnue au fait dialogique.

Ce que, à l'orée de la réception de Bakhtine en France, J. Kristeva (1970 : 76) désignait comme manque - « une théorie de la signification qui aurait besoin d'une théorie du sujet » - apparaît plus précisément comme une approche de la subjectivité penchant du côté de la conscience et de l'intentionnalité, « en

du préconstruit (AD) et de la polyphonie ( $c f .2 .11$ (iii) ci dessous). Sur la distinction à faire entre « marques » et « indices », voir ci-dessous chap. 11 Remarque 1 p. 435.

41 Je m'efforce ici d'éviter la formulation de cette problématique en termes de « double dialogisme interdiscursif /interlocutif », dont la séduisante commodité (à laquelle je n'éviterai pas de céder çà et là...) ne doit pas masquer l'impropriété syncrétique ( $c f$. ci-dessous) au regard des divergences théoriques de fond qui, en deçà de ce qui les rassemble, opposent « dialogisme » et « interdiscours".

42 Voir les pertinentes remarques de J. Bres (2008) sur la fréquence de «l'oubli de Bakhtine » et de l'épaisseur « verticale » du dialogisme « interdiscursif », dans les approches de l'interaction « dialogale».

43 Cette question du « Dire pour l'autre dans le déjà-dit » (Authier-Revuz 2012) est ainsi au cœur de Sitri (2003), des recherches de J. Bres (1999, 2008), de celles de P. Von Munchow (2011, 2012), et on le voit émerger au sein de la « Dialogue analysis », dont le colloque (IADA 2009) invite dans son intitulé à croiser « intertextualité » et « interlocution », cf. Lorda Mur (2012).

44 Comme déjà dans Authier-Revuz (1995/2012), à propos de la dimension du déjà-dit des formes de «non-coïncidence interlocutive " (le « dialogisme interlocutif large » d'un comme vous avez l'habitude de dire vs comme vous venez de dire) ou de la spécification interlocutive des formes de renvoi à un extérieur interdiscursif (comme on dit chez vous, dans votre parti, ...) (cf. ci-dessous chap. 11.2.3.1, p. 443 les incidents interlocutifs sur fond de déjà-dit du dire). 
manque » des moyens théoriques qui lui permettraient de penser ce qui du dialogisme foncier, centralement affirmé, échappe à cette conscience intentionnelle. Au-delà du rappel du rejet radical du freudisme par Voloshinov ${ }^{45}$, les travaux récents de Sériot (2007 : 45-46) soulignent, solidaires de leurs ancrages respectifs, psycho-sociologique pour Voloshinov et dans « une approche personnaliste de la responsabilité » pour Bakhtine, une même absence de la dimension d'un inconscient, divisant le sujet.

De fait, l'appréhension du mouvement du discours conjoignant la visée d'une « orientation sur l'objet » et l'emprise de l'extérieur discursif sur le discours, oscille, non problématisée, au gré des formulations et des métaphores, entre choix intentionnels (« le locuteur cherche à orienter son discours avec son point de vue [...], il construit son énoncé [...] $»^{46}$ ) et processus discursifs (c'est « le discours » ou « l'énoncé » qui rencontre, traverse, trouve, touche, fusionne, etc. le matériau du déjà-dit) ${ }^{47}$. Et, essentielles quant au rapport du sujet au discours et au sens, les questions de la (non-) maîtrise et de la (non-)représentabilité affleurent constamment à travers les dimensions reconnues de contrainte (le « ne peut pas ne pas » cher à Bakhtine) et d'extériorité (le discours qui « se joue en dehors de l'auteur »), sans accéder pour autant à une théorisation explicite.

À ce qui - déficit opératoire ou impasse théorique - contrarie la perspective d'une effective mise en regard, pour un discours, un texte, de ce qu'il représente comme autre (RDA) avec ce qu'on peut approcher de son extérieur constitutif, c'est dans les élaborations de Pêcheux et le recours à l'approche post-freudienne d'un sujet divisé, qu'on tentera de trouver des réponses articulant les deux plans du constitutif et du représenté, sans perdre la richesse du « tissage » qu'en propose le dialogisme bakhtinien.

45 Voloshinov (1927), $c f$. « (Bakhtine) et Freud » in Houdebine (1977 : 129-223) et Authier-Revuz (1995/2012: 102-104).

46 Bakhtine 1975 : 105.

47 Ce va-et-vient entre processus de discours et conscience d'un locuteur se manifestant à l'extrême dans des formulations telles que (à propos de discours autres ne se prêtant pas aisément à leur assimilation) :« C'est comme si, hors de la volonté du locuteur, ils se mettaient "entre guillemets" » (Bakhtine 1975 : 115) où ce serait « comme si » le discours accédait à la conscience représentante que suppose le guillemet... 


\section{2 « Ça parle » toujours « ailleurs, avant et indépendam- ment ${ }^{48}$ : l'interdiscours au principe du discours}

L'ensemble - conceptuel et méthodologique - produit dans le cadre de ce que D. Maldidier (1990) appelle "l'aventure théorique du discours », l'AD telle qu'elle s'est développée autour de Michel Pêcheux à partir des années $60^{49}$, a été, et demeure aujourd'hui, à mes yeux, un appui déterminant pour poser - en " fond » de la description des formes de RDA, qui représentent de l'autre, de l'ailleurs, dans le dire - ce que j’ai appelé « l'hétérogénéité (interdiscursive) constitutive».

Contrairement à la rencontre « inévitable » du dialogisme avec sa problématique générale de "l'autre dans l'un » et son attention aigüe aux formes de sa représentation, pour qui, dans les années 70 , s'intéressait aux formes du « dis-

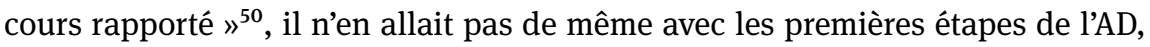
marquées à la fois par une prédilection pour l'homogène et un désintérêt explicite pour les formes de l'énonciation... En revanche, dès lors que, dans son constant mouvement de transformation, elle fait place $\left(\mathrm{AD}_{3}\right)^{51}$ au réel de l'hétérogène et à la consistance des formes de l'énonciation, cette approche du « discours comme produit de l'interdiscours » va étayer (à partir de Authier 1982, 1984) la notion d'hétérogénéité constitutive/représentée, à côté de celle de dialogisation interne du discours, dont l'AD partage la pensée de l'historicité et de l'extériorité interne

48 (Pêcheux 1975 : 147), le « ça parle » étant repris à Culioli reprenant Lacan.

49 Je ne retiens pas, pour ce mouvement, l'étiquette d' 'école française d'analyse de discours » proposée, a posteriori, dans DAD (2002: 201-202) ; elle apparaît, notamment, problématique quant à la place de travaux tels que ceux de J. Dubois, B. Gardin, J.B. Marcellesi... : sont-ils exclus, alors même que incontestablement ils relèvent d'une analyse de discours, spécifiquement ancrée dans le paysage intellectuel et politique français des mêmes années, ou, aussi indûment, assimilés à la démarche de Pêcheux, en dépit des clivages vifs qui les séparent ? Paveau et Rosier (2005) reviennent, citant l'analyse critique qu'en fait Courtine, sur les difficultés de cette appellation. Il va de soi que je souscris encore moins au congédiement - en tant que « progressivement marginalisé à partir des années 80 » (DAD 2002: 202) et « appartenant à l'histoire des idées » (Maingueneau 1995) - d'un courant qui, au rebours (plus encore aujourd'hui que trente ans en amont) des évidences consensuelles des sujets " gestionnaires » de leur dire et de leur sens, nourrit nombre de travaux, et en tout cas, en ce qui concerne ce travail, la pensée du discours et de son « hétérogénéité constitutive ».

50 Voloshinov (1929), premières traductions anglaises en 1973, française en 1971, dont la lecture récente se manifeste ponctuellement dans Authier et Meunier (1977: 63), et dans Authier (1978 : $51,74,82)$.

51 Cf. Pêcheux en 1983 « Analyse de discours : trois époques » (1990: 295-302), voir note 71 ci-dessous. 
au dire, en apportant, dans ce qui les différencie, des éléments, précieux, d'affermissement théorique et d'opérativité descriptive ${ }^{52}$.

Emergeant dans la conjoncture théorique des années 60 - rangée, sommairement, sous la bannière «Marx, Freud, Saussure »- le concept de " discours » mis en œuvre dans l’AD croise les questions de la langue, du sujet et du sens. Du parcours de l'AD, je rappellerai schématiquement ${ }^{53}$ (1) à travers les constantes de sa démarche, ce qui en fait la force aux plans du positionnement théorique et de l'investigation descriptive, avant (2) de marquer, dans l'évolution de leur mise en œuvre (périodisée en «trois époques » par Pêcheux), celles des reconfigurations qui permettent la prise en compte de l'hétérogène représenté de la RDA.

\subsection{Choix fondamentaux d'une démarche en évolution}

D’un bout à l'autre (souligne D. Maldidier, 1990 : 89) ce que [M. Pêcheux] a théorisé sous le nom de discours est le rappel de quelques idées aussi simples qu'insupportables : le sujet n'est pas à la source du sens ; le sens se forme dans l'histoire à travers le travail de la mémoire, l'incessante reprise du déjà-dit ; le sens peut être traqué, il échappe toujours.

Le lieu du « discours », en effet, - quelles que soient les catégories à travers lesquelles est pensé son " ordre » : des «formations discursives » homogènes initiales aux « réseaux » ou « espaces » de « mémoire discursive », en passant par les

52 C'est d'abord par les échanges et discussions, nourries par la générosité intellectuelle de M. Pêcheux que, dans le cadre d'entreprises diverses (section de linguistique du « Centre d'Etudes et de Recherches Marxistes », 1977-1980 ; colloque « Matérialités discursives » (Conein et al. 1981) ; dernier groupe de recherche, ADELA (Analyse de Discours et Lecture d'Archive, RCP du CNRS), 1981-1983, animé par M. Pêcheux, dont D. Maldidier (1990) retrace précisément l'activité) j’ai bénéficié de la profondeur et de la richesse d'une pensée exigeante et ouverte qui, de fait - qu'elle soit citée ou non - irrigue, souvent croisée à celle de Bakhtine selon des modalités diverses et complexes, tant de travaux actuels, dès lors qu'ils ne tournent pas le dos - cédant complètement à l'idéalisme des mirages communicationnels, interactifs, pragmatiques, d'échanges entre sujets stratèges du sens - à la prise en compte de la détermination « (inter)discursive » du sens.

53 Pour un parcours moins cavalier, relevant de la même perspective, je renvoie aux pages qui y sont consacrées dans Authier-Revuz (1995/2012) : " Théorie discursive du sens de Pêcheux ", p. 236-238, et " L'hétérogène en analyse de discours (AD) », p. 245-257; pour des traitements d'ensemble de cette AD, outre aux présentations de Maingueneau (1976, 1987, 1991) qui en ont activement accompagné et transmis le parcours, à la précieuse introduction donnée par D. Maldidier « (Re)lire Michel Pêcheux aujourd'hui » (p. 7-91) à son recueil de textes de Pêcheux (1990), paru sous le titre « L'inquiétude du discours »; et plus récemment, à la synthèse de F. Mazière (Mazière 2006) et à des mises au point (Paveau 2008, 2010, Paveau et Rosier 2005), contre des recouvrements ou confusions. 
redéfinitions du concept « clef de voûte » de l'interdiscours - détermine la forme et le sens des énoncés observables.

Ainsi, au fil des reconfigurations de l'AD, est-ce à travers une série de couples de notions articulant les deux plans de l'énoncé et du lieu discursif dont il procède, que par leur mise en rapport, réglée, sont appréhendés les processus discursifs, par lesquels le fait observable d'une linéarité énoncée ici-maintenant dans sa singularité (I) apparaît comme le produit d'une discursivité extérieure « formée » ailleurs, avant et indépendamment (II). Autour du couple central intradiscours/ interdiscours se déploient : surface (discursive) et extérieur spécifique ${ }^{54}$, dedans et « ailleurs », actuel et antérieur, séquence et «formation discursive », horizontal et « vertical », linéarité et épaisseur, fil du discours et domaine, espace ou réseau de mémoire, etc..., les espaces discursifs du (II) n'étant pas pensés comme les « contextes » des observables du (I), mais comme ce qui, à leur insu, les « détermine », les « régit », s'y « inscrit », s’y dépose, s'y impose sur le mode d'une extériorité interne ${ }^{55}$.

S'en trouvent, au plan du sens, défaits - déportés qu'ils sont, hors d'euxmêmes, par cette instance extérieure - le texte (le dire) comme clôture, le sujet comme source, origine.

\subsubsection{Un socle stable}

Trois points assurent le « socle » permanent des étapes de cette démarche :

\section{(i) La contestation du sujet psychologique, source intentionnelle d'une parole} dont il serait à même de se représenter le sens

Derrière la variation des modalités sous lesquelles est appréhendée « l'objectivité matérielle » de l'extérieur discursif où se déterminent le dire et son sens, demeure : que c'est à l'insu du sujet, dissimulée sous "l'évidence » d'un sens transparent dont il serait la source intentionnelle que se produit l'emprise du dehors sur le dedans ; que nul n'est à même d'accéder à cet avant-ailleurs discursif qui « cause » (dans) son dire; et que, par là, de ce dire dont la maîtrise lui

54 Cf. Pêcheux dans « Analyse automatique du discours » (1969) [in (1990: 130)].

55 C'est ce que - après avoir noté comment « tout discours dominé est tissé de discours dominants qui lui sont intégrés », comment " le savoir antérieur s'inscrit dans la construction d'une connaissance et se répète à travers des formes de langue »- souligne F. Mazière (2005: 58) : « Autrement dit, hétérogénéités et antériorités de l'interdiscours s'inscrivent à l'intérieur même de l'intradiscours, elles n'en constituent pas le contexte.». 
est dérobée, le sens est, pour le sujet qui l'énonce, irreprésentable $e^{56}$. A travers les déplacements de fond qui l'affectent ( $c f$. ci-dessous 2.1.2) ce point se distingue nettement des inflexions « conscientisantes » du dialogisme.

\section{(ii) Une pensée du discours arrimée au fait de la langue}

La reconnaissance d'un ordre du discours s'oppose, d'emblée, à la " parole », pensée comme rapport direct d'un sujet à la langue dans sa visée du sens : cet ordre langagier, historiquement structuré - dans le double sillage " structuraliste » de Foucault et d'Althusser ${ }^{57}$ - ne vient en aucune façon chez Pêcheux se substituer à l'ordre de la langue. L'affirmation de la matérialité de la langue saussurienne, comme système différentiel de formes, reposant sur la "valeur » est une des positions intangibles de l'AD à travers toutes ses configurations ${ }^{58}$ : cet « arrimage » au linguistique ${ }^{59}$ inscrit au cœur de l'AD (tant au plan théorique qu'à celui des procédures descriptives attachées aux formes de langue) la problématique de l'articulation de cette double systématicité - celle d' ' un ordre propre [du discours], distinct de la matérialité de la langue » mais qui «se réalise dans la langue $»^{60}$.

Modalité de la " prise » de l'interdiscours dans l'intradiscours, le « préconstruit » comme forme de langue (relative déterminative, nominalisation, focalisation.... ${ }^{61}$ propre à accueillir sur le « fil du discours » le préasserté ailleurs,

56 Pensons à l'expérience de certains « dialogues de sourds » entre interlocuteurs disposés pourtant à l'échange : s'y joue l'impossibilité où ils se trouvent de s'extraire du discours dans lequel « baigne » leur parole, pour « mettre sur le tapis », en débat - c'est-à-dire objectiver, expliciter ce qui s'impose, c'est-à-dire se dérobe, à eux sous le mode de l'évidence. On ne peut pas parler de ce par quoi "on est parlé". Pour le dire autrement, on ne "rencontre" pas dans sa parole ce qui y va de soi - ce qui questionne quant à ses limites la pertinence de la métaphore dialogique de la rencontre-réponse entre dire et déjà dit.

57 Cf. notamment la théorie marxiste des idéologies d'Althusser (1970) et « L'archéologie du savoir » de Foucault (1969), faisant peu de place à la langue ; ceci contrairement à Lacan, autre référence de Pêcheux, qui convoquée d'emblée, en rapport avec la place faite à la langue, n'est pas prise en compte au plan du sujet avant l'AD $\mathrm{D}_{3}$.

58 Présente centralement dès l'article réaffirmant (Haroche et al. 1971) « la coupure saussurienne » comme condition pour aborder ce qui - la sémantique - excède le champ de la langue (cf. Maldidier 1990 : 19-23), elle se manifestera dans la critique répétée ( $c f$. par exemple Pêcheux 1982) des prétentions, analysées comme des régressions pré-théoriques, à « dépasser » Saussure, en rejetant la langue du côté des artefacts normatifs.

59 Selon l'heureuse formule de D. Maldidier (1990: 13).

60 Cf. Courtine (1981b) cité in Maingueneau (1987: 12).

61 Cf. par exemple Grésillon (1975) pour les relatives, Sériot (1986) pour les nominalisations. 
avant..., incarne exemplairement cette articulation des "processus discursifs » à une « base linguistique ». La consistance également conférée au discours et à la langue tranche évidemment avec le rejet offensif de Saussure par Voloshinov vidant le concept de langue au profit d'un «tout-social »-comme avec les accueils enthousiastes qui ont pu être faits de Marxisme et philosophie du langage au titre d'une refondation anti-saussurienne de la linguistique ${ }^{62}$. Moins incompatible avec la position spécifiquement bakhtinienne, l'AD se démarque cependant nettement, sur cette question, par le souci foncier d'articuler les deux « ordres », de la langue et du discours, du mode sur lequel Bakhtine, concédant la légitimité d'une linguistique, en « détache » quelque peu le trans- ou le métalinguistique, espace du dialogisme.

\section{(iii) dimension opératoire revendiquée théoriquement}

Il n'est pas question, chez ce philosophe, de s'en tenir à une conceptualisation articulant histoire, sujet, sens, mais de construire explicitement les « dispositifs » - terme cher à M. Pêcheux - mettant les propositions théoriques à l'épreuve des matérialités langagières ${ }^{63}$.

Ainsi l'affirmation centrale, d'un

primat de l'interdiscours comme affectant tout discours d'une hétérogénéité par rapport à lui même, dont il appartient à l'AD [...] de repérer les traces. (Courtine 1981 : 31)

associe-t-elle conception théorique et visée descriptive : consubstantielle à la démarche de l'AD est la pratique - solidairement théorique et méthodologique de la mise en rapport des deux plans de la matérialité discursive - celui de la linéarité directement observable de l'événement singulier de dire et celui de l'épaisseur stratifiée de déjà-dit d'où procède le premier. Au-delà du « principe » de l'extériorité qui, interne au dire, le « déclôture », l’AD est une tentative pour appréhender - « sur pièces » - quelque chose de son « comment », en donnant corps, dans des analyses concrètes, au jeu, dans un discours (I) de son extérieur (II).

62 Cf. Gardin (1978) par exemple. Sur la vivacité de ce débat dans le champ marxiste des années 1970, $c f$. Maldidier (1990: 49-53).

$63 C f$., par exemple, à propos des vertus, en $\mathrm{AD}$, de l'informatique - mise à l'œuvre, tout au long du parcours de l'AD, sous des formes diverses - le fait qu'elle « exige des analystes de discours une construction explicite de leurs procédures de description, ce qui est la pierre de touche de leur consistance d'objets théoriques. » (Pêcheux et Marandin 1984). Cf. aussi la réflexion de P. Henry (1995) sur le lien, dans les conceptions épistémologiques de Pêcheux, entre « nature conceptuelle et expérimentale » de l'objet scientifique et sur l'accent mis sur « l'instrument ». 
Et l'histoire de l'AD est, indissolublement, celle de ses renouvellements théoriques et procéduraux : aux conceptions successives de l'extériorité discursive et de son emprise sur les énoncés, répondent, au plan des procédures de construction de cette mise en rapport, les modes successifs selon lesquels, solidairement,

- (a) elle construit en corpus, analysable, quelque chose du dit ailleurs, avant et indépendamment, dans lequel, au plan (II), se produit et/ou se reçoit une séquence,

- $\quad$ et (b) elle analyse, au plan (I), les formes de présence sur la chaîne de cet extérieur discursif.

Remarque 3 : Évolution conjointe des conceptions théoriques et des procédures descriptives. Pièce maîtresse du dispositif de l'AD $^{64}$, la construction du corpus (a), au départ [1] synchronique et homogène (correspondant à la compacité insulaire des « machines » structurelles de la première $\mathrm{AD}$ ), intègrera [2], avec la notion d'interdiscours, l'hétérogène de la contradiction, et de la stratification diachronique ${ }^{65}$, avant que le corpus ne soit [3] étendu à de très vastes étendues discursives susceptibles d'être " balayées » par des procédures informatisées, et adapté évolutivement, en « états de corpus ", à une progression de l'analyse, comme accès au « réseau de formulations » ou « corps socio-historique de traces discursives » constituant « l'espace de mémoire d'une séquence $»^{66}$.

Cependant que, parallèlement, l'analyse (b) des chaînes passera par : [1] une pratique délinéarisante de réduction à l'équivalence des spécificités de surface permettant de remonter vers leur « principe structural », avant [2] de privilégier, à côté de strictes reprises de segments entre (I) et (II), les formes linguistiques d'accueil du déjà-dit que sont « préconstruit, discours transverse, énoncé divisé... », et [3] de viser la construction automatique des associations produites au fil d'un discours « plongé » dans l'espace de mémoire d'une « lecture ».

Dans ce cheminement, la trilogie interdiscours-intradiscours-préconstruit apparaît comme emblématique de l'AD - par la netteté avec laquelle s'y noue l'ensemble des choix sur lesquels, spécifiquement, elle repose. C’est ce qui se dégage de la mise en regard de la notion de préconstruit avec celle - répondant au même type de formes observables dans la linéarité, telles : nominalisations, interrogations partielles... - de présupposition, mise en œuvre par O. Ducrot.

64 Je renverrai désormais par [1], [2], [3] aux étapes de la périodisation proposée par M. Pêcheux en 1983 [in (1990 : 295-302)], à laquelle j’associe la succession des textes choisis par D. Maldidier (en dépit d'une différence dans le découpage qu'elle effectue) :

[1] $\mathrm{AD}_{1}$ : centrée sur l' "Analyse automatique du discours » (1969), textes I à III ;

[2] $\mathrm{AD}_{2}$ : allant des « Vérités de la Palice » (1975) à 1980, textes IV à VII ;

[3] $\mathrm{AD}_{3}$ : à partir du colloque «Matérialités Discursives » de 1980, textes VIII à XI.

65 Courtine (1981).

66 Pêcheux 1983 in (1990: 301). 
Dans le statut conféré, par rapport à une assertion (a) et une séquence (b) comportant sa nominalisation,

(a) le climat se réchauffe

(b) le réchauffement climatique impose de modifier nos modes de vie

le clivage entre les deux concepts apparaît sur chacun des points (i, ii, iii) dégagés ci-dessus. Ainsi l'acte de présupposer engage-t-il l'intention d'un sujet psychologique [point (i)], ayant recours, stratégiquement, pour soustraire ce qu'il dit - donné pour évident - à la contestation, aux ressources d'implicitation offertes par la seule langue [point (ii)]. Là où le préconstruit, trace inscrite dans le dire d'une extériorité, n'engage pas l'intentionnalité [point (i)] (qui, certes, peut s'en emparer), mais implique la prise en compte de deux ordres, de la langue et du discours [point (ii)] et leur mise en rapport [point (iii)]. Dans la confrontation préconstruit/présupposition ${ }^{67}$ se manifeste exemplairement la divergence entre une « sémantique (intra)linguistique », croisant la langue et l'intentionnalité communicative, et le projet de « sémantique discursive » de Pêcheux.

Sur ces choix ( $i$, ii, iii), posés comme formant le « socle » théorico-méthodologique stable du trajet de l'AD, deux remarques :

- La première touche aux divergences entre $A D$ et dialogisme qui, dans leur traitement d'une extériorité discursive reconnue comme interne au dire, ne sont pas mineures : au-delà de la place, différente, faite à la langue, l'opposition entre, d'un côté, la détermination d'un dire, à son insu, par un extérieur discursif et, de l'autre, les " rencontres » de son " cheminement » dans le déjà-dit, doublée par celle, solidaire, de la visée d'une saisie de « traces » de présence insue pour le premier (AD), là où le second (dialogisme) met l'accent sur les formes de représentation, incite à interroger le syncrétisme, tentant et répandu ${ }^{68}$, de formulations comme « dialogisme interdiscursif » ou « interdiscursivité bakhtinienne »... : la double filiation qu'elles reflètent appellerait, cette association n'allant pas de soi, une explicitation des modalités sur lesquelles elle est, diversement, opérée - dont on peut penser que, de façon dominante, elle relève d'un mouvement de « bakhtinisation » de l'AD ${ }^{69}$ où le «ça parle » tend à se dissoudre en « rencontres » dialogiques... Rappelons ( $c f$. note 63) à quel point la métaphore du « dialogue » - avec les deux instances symétriques qu'il suppose - envisageable pour saisir le rapport d'un Discours

67 Qui est au cœur de Henry (1977), suivi de la « réponse » de Ducrot. Sur le rapport entre les deux notions, $c f$. Maldidier (1990 : 26-27), Paveau (2006 : chap. 2).

68 Tendance dont je ne m'exclus pas, $c f$. Authier-Revuz (1995/2012 : 24).

69 Selon l'heureuse formulation - critique - de M.A. Paveau (2008), $c f$. note 60. 
à l'altérité discursive qu'il reconnaît comme telle, notamment en la représentant en lui-même, est radicalement inadéquate pour le mode sur lequel un discours dépend de l'extériorité discursive dans et de laquelle il se « constitue » : on ne « dialogue » pas avec son interdiscours.

De l'interdiscours de l'AD au « dialogisme interdiscursif », le déplacement apparaît comme celui d'une " psychologisation », ramenant tendanciellement l'impensé, pour le sujet, de ce qui détermine son dire, vers la (quasi) conscience des rapports du dire avec l'environnement d'autres dires. Sous les figures de ce à quoi on " emprunte ", à quoi on " répond " ${ }^{70}$, l'interdiscours semble avoir rompu les amarres avec l’AD qui - insiste D. Maldidier (1990 : 43) -, conceptualisant le « ça parle toujours ailleurs, avant et indépendamment », « n'est pas la désignation banale des discours qui ont existé ».

- La seconde pour souligner que les (trois) choix fondamentaux dégagés ci-dessus, au niveau de généralité qui permet, en deçà des transformations qu'à connues l'AD, d'en faire le socle intangible, n'offrent pas, en eux-mêmes, un cadre à une saisie de l'articulation des hétérogènes constitutifs et représentés : la forme que prend leur mise en œuvre dans la première $\mathrm{AD}$ est même radicalement inadéquate à une approche de la RDA, comme couche représentée d'une extériorité discursive interne.

\subsubsection{Ouvertures sur l'hétérogène et le fil énonciatif du discours}

L'AD n'offrira un cadre propice à une problématique de la RDA qu'au terme de mutations profondes ${ }^{71}$, ouvrant l'AD - au risque de sa déstabilisation - sur les dimensions, forcloses au départ, (i) de l'hétérogène, (ii) de la consistance propre d'un « fil du discours » où s'inscrit l'énonciation, solidairement (iii) d'un retour critique sur la conception du sujet, restauré - non dans sa maîtrise - mais dans sa consistance (singulière) de division.

70 Ainsi J.M. Adam (2006) illustre-t-il ce qu'il caractérise chez Peytard (1995) comme un « glissement de l'interdiscours vers le dialogisme de Bakhtine », par sa définition (déjà relevée, dans la même perspective dans Paveau-Rosier 2005 : 11) du « tiers-parlant » comme " ensemble indéfini d'énoncés prêtés à des énonciateurs sous les espèces de: “les gens disent que...”, “on dit que...”, "on prétend que...”. [...] Ces énoncés [doxiques] appartiennent à la masse interdiscursive à laquelle empruntent les agents de l'échange verbal pour “nourrir” leurs propos » (1995:121). Citons encore cette définition: « le dialogisme interdiscursif structure fondamentalement tout texte en ce que celui-ci est obligatoirement réponse (Bakhtine 1952/1979/1984: 298-299) à des textes antérieurs ». (Bres-Nowakowska 2006: 25-26).

71 Sur ces points je renvoie à D. Maldidier (1990) ; Pêcheux 1983 in (1990: 295-302) ; Authier-Revuz (1995/2012 : 95-96 et 245-253). 


\section{(i) Vers l'hétérogénéité de "l'ailleurs-avant"}

Le trajet de l'AD, dans sa conceptualisation de l'extériorité discursive déterminant le discours, va d'un " primat du même » $\left(\mathrm{AD}_{1}\right)$ à celui de « l'autre sur le même » $\left(\mathrm{AD}_{3}\right)^{72}$.

De [1] « l'insularité » des « machines » discursives homogènes de « l'analyse automatique du discours » (1969), on passe en [2] à la prise en compte, dans la mise en rapport de « formations discursives » dans le « tout complexe » de l'interdiscours, de faits, d' « intrications », d'empiètements entre ces unités qui, faisant jouer de l'autre, les « divisent » et « déclôturent ».

Se conjuguent, dans ce mouvement, la réflexion théorique sur la catégorie de l'idéologie comme « non identique à soi-même et n'existant que sous la modalité de la division ${ }^{73}$, et les études des historiens confrontant leur quête "dans le corps complexe des discours [d']éléments simples (discours bourgeois/discours féodal ; discours jacobin/discours sans-culotte) » au réel d'une discursivité où ne se rencontrent « qu'intrication des stratégies discursives d'affrontement ou d'alliance. » (Guilhaumou et Maldidier, 1979). L'étude de Courtine (1981), consacrée $\mathrm{au}$ « discours communiste adressé aux chrétiens », théorise et pratique, aux plans inter- et intra-discursifs, cette entrée de l'hétérogène sous les espèces de la contradiction (communiste /chrétien) et du décalage diachronique, dans la construction du corpus et dans les « effets de mémoire » jouant, par exemple, dans la séquence de « L'appel adressé aux chrétiens » de G. Marchais en $1976^{74}$.

Le mouvement aboutit en [3] à la mise en cause d'une approche « topographique » de l'(inter)discursivité (et par là même à la notion même de formation discursive qui y ancrait sa consistance), dans le constat que c'est « sans frontière repérable » que « la séquence est constituée - traversée par des discours venus d'ailleurs ${ }^{75}$, menant à une reconfiguration de l'interdiscours en " espace de

72 Pour reprendre les formulations de Pêcheux 1983 in (1990: 297, 299).

73 M. Pêcheux « Remontons de Foucault à Spinoza » en 1977, in (1990 : 245 sq.)

74 Exemples encore ( $c f$. in Authier-Revuz 1995/2012: 250, 253) de mise au jour systématique dans un discours - soviétique pour Sériot $(1985,1986 \mathrm{a}, \mathrm{b})$, « nationalitaire algérien » pour Ouamara (1983), repris en partie dans (1986) - d'un hétérogène jouant dans les nominalisations pour le premier, les formes de « déconstruction » du discours colonial pour le second. La stratification diachronique des corpus est au cœur des travaux - connexes à l'AD - sur la « circulation » discursive dans les médias (Moirand, 2007) ou l'émergence - et les avatars - d'une «formule » (Krieg, 2003).

75 Madidier (1990 : 78) reprenant un manuscrit de Pêcheux (des années 1982-83). Le colloque Matérialités discursives (Conein et al., 1981) marque le tournant, en AD, de cette ouverture sur l'hétérogénéité des « espaces de mémoire »: au retour critique initial de Courtine et Marandin sur le « ratage de l'hétérogène » (p. 24), répond, dans le bilan signé des organisateurs (p. 99) l’in- 
mémoire d'un corps socio-historique de traces discursives ${ }^{76}$ dans lequel se constitue une séquence dont la « lecture », par une AD repensée en discipline interprétative, passera par sa mise en rapport avec des « réseaux de mémoire ».

Ainsi, le dernier texte de Pêcheux ${ }^{77}$ esquisse-t-il, à partir de " l'opacité » de l'énoncé « On a gagné ! » du soir de la victoire de F. Mitterrand en 1981, la dispersion des réseaux de mémoire dans lesquels il se trouve résonner. Et, dans des développements récents de l'AD, on voit que s'impose, avec son « impossible clôture $»^{78}$, le caractère hétérogène de la mémoire discursive à l'œuvre dans un discours : la présentation d'une recherche collective en cours par Ph. Schepens (2012), par exemple, illustre, dès son titre « Prolégomènes à l'analyse d'un journal écrit sous l'occupation : Qui parle ? », comment la « plongée » d'un texte - ici, le journal intime d'une jeune fille du Doubs durant cette période - dans la mémoire, immense, hétérogène et extensible ${ }^{79}$ - que les techniques de numérisation permettent de constituer - est le préalable à une " lecture » de ce texte, pensée comme mise en rapport réglé de l'un qui s'énonce avec l'autre de l'extériorité discursive, par laquelle ce dire personnel se révèle « lieu [...] d'entrechocs entre discours constitués ou institutionnels et parole vivante qui fraie son chemin »; ou les réflexions sur lesquelles débouche l'étude d'écrits de «Signalement d'enfants en danger ${ }^{80}$ soulignent le caractère hétérogène et ouvert du corpus de mémoire que requiert l'analyse de ce discours où se tissent textes de lois, guides de rédaction, discours médiatiques, psychologiques, psychanalytiques...

\section{(ii) Entrée du fil, énonciatif, du discours}

La solidarité que note P. Henry (1977 : 154) entre énonciation et linéarité :

[...] dans le discours commun, ce qui soutient cette identité [du sujet de l'énonciation présent sous le sujet de l'énoncé] c'est la linéarité du discours.

apparaît dans le mode conjoint sur lequel leur traitement évolue radicalement sur le trajet de l'AD.

sistance sur « la frontière absente » (titre de ce bilan), ou « inassignable » (p. 202) dans le mode - non « localisable » - où « du même est pris dans l'autre » (p. 202).

76 Pêcheux 1983 in $(1990$ : 301).

77 «Le Discours : structure ou événement », in (1990 : 306-313).

78 Cf. Moirand (2004).

79 Où cohabitent déjà, par exemple, 200 tracts de la résistance et une collection complète du quotidien régional.

80 Émanant des services sociaux, $c f$. Cislaru et Sitri (2012). 
La contestation - inhérente à l'AD - du sujet maître de sa parole, prend, en effet, dans la première étape [1] la forme d'un égal désintérêt - voire d'une suspicion $^{81}$ - vis à vis de la matérialité du fil du discours et des marques de l'énonciation : c'est conjointement qu'ils sont écartés comme relevant de «l'espace imaginaire » offert aux déplacements du sujet parlant ${ }^{82}$, sans autre consistance que celle d'un leurre, sorte d' « écume » à traverser pour accéder - par des pratiques de « désuperficialisation » permettant de « remonter » des énoncés concrets jusqu'à leur « principe structural ${ }^{83}-\mathrm{au}$ " ça parle » des processus discursifs, cause véritable du dire. Ainsi, revenant, en 1983, sur cette première approche du discours, Pêcheux (1990 : 300) note-t-il que, dans sa logique, « le registre de l'énonciation et des contraintes de séquentialité lui demeurait opaque $»^{84}$.

$\mathrm{Si}$, dans un deuxième temps [2], l'attention aux formes syntaxiques - du préconstruit notamment - est manifeste, c'est dans le cadre ignorant la dimension proprement énonciative - « a-énonciatif » pourrait-on dire - de l'articulation interdiscours/intradiscours, comme trace du premier dans le second.

Ainsi faut-il attendre le colloque Matérialités discursives, ouvrant la dernière période $^{85}$ [3] de l'AD, pour que l'attention se porte enfin, comme sur un objet légitime, sur « cette matérialité même de l'activité énonciative » dont Culioli notait, pertinemment, le « peu de cas [qu'en avaient fait] les spécialistes d'analyse du discours ${ }^{86}$. Diverses contributions s'y attachent ainsi aux formes d'inscription de la subjectivité affectant le déroulement de la linéarité : les « règles » de langue pensées comme « espace de jeu » pour l'écriture, les « effets de déliaison » (par rapport aux structures d'enchâssement) des juxtapositions d'énoncés, interruptions, phrases nominales, les ajouts de l'incise et les « trous » de l'ellipse, les « arrêts sur mots » des guillemets, suspendant de leur dédoublement méta-énon-

81 Parente de celle de Kuentz (1972a: 22, 27) à l'égard de toute notion - « parole », « énonciation »... - susceptible de servir une « opération de sauvetage » de « l'autonomie du sujet parlant ». 82 «L'espace subjectif de l'énonciation » y est entendu, en effet (et par là, dans cette première étape, « dévalorisé »), comme « espace imaginaire qui assure au sujet parlant ses déplacements à l'intérieur du reformulable de sorte qu'il fait retour sur ce qu'il formule et s'y reconnaît. » (Pêcheux et Fuchs 1975 : 21) - espace où s'assure, pour le sujet, une position illusoire d'extériorité par rapport à son discours.

83 Sur cette procédure de délinéarisation des chaînes observables, réduites à l'équivalence par transformations harrissiennes « inverses » de dé-passivation, dé-nominalisation, dé-subordination... voir la présentation de Maingueneau (1976 : 65-82).

84 Gadet et al. (1995) reviennent sur la « place du pauvre accordée à Benveniste » dans une AD « passant à côté de l'énonciation » en 1969.

85 Annoncée, sur ce point, dans l’analyse par Marandin (1979) du « fil » du « discours français sur la Chine ».

86 Intervention à la table ronde finale du colloque (Conein et al., 1981 : 185). 
ciatif le cours du dire... ${ }^{87}$, focalisent l'attention sur - événements, émergences, accidents... - ce qui survient, advient, dans le cours d'un dire à la « consistance retrouvée », sans que cela implique, en aucune façon, un sujet « restauré » dans sa maîtrise.

Dans ce mouvement, l'AD [3] s'ouvre à la dimension, métalangagière, de la représentation du dire - dont relève notre RDA - c'est-à-dire, dans une approche de l'impensé du dire, aux formes qui marquent que ce dire, pour autant, se pense. Le principe d'une articulation entre les deux plans des hétérogénéités « montrée/ constitutive » trouve dans le cadre d'ADELA ${ }^{88}$ - pensée comme façon de « revenir dans des termes nouveaux et opérationnels vers le jeu de l'interdiscours dans l'intradiscours » (Maldidier 1990 : 78) - un lieu privilégié de mise au travail.

Le jeu dans le discours des « formes linguistiques discursives du discours autre " peut alors être envisagé dans une double visée descriptive : celle des marques de la « mise en scène par le sujet » du « discours d'un autre » et « en même temps » celle des traces de "l'instance d'un 'ailleurs' interdiscursif [...] en deçà de tout auto-contrôle fonctionnel ${ }^{89}$. On revient ci-dessous (chap. 11) sur la mise en rapport de ces deux plans où l'extérieur discursif joue dans le dire : sa prise en compte, en effet, conditionne l'approche de la fonction, dans le dire, de la RDA - ce que celle-ci « représente » de l'ailleurs se profilant sur fond de ce qui en est « présent ».

\section{(iii) Du «non-sujet » au sujet divisé : un déplacement de fond}

L'évolution sur les plans de l'hétérogène (i), du « fil » et des formes énonciatives (ii), est inséparable d'un déplacement de fond sur la question du sujet.

Le trajet de l'AD s'inscrit, en effet, dans l'écart flagrant entre une visée initiale [1] « [d']esquisser une théorie non-subjective de ce qu'on appelle aujourd'hui énonciation »

et l'ouverture [3] sur « [la] question cruciale du sujet énonciateur, dans la parole, l'écoute et la lecture. ${ }^{90}$

87 Traités dans les contributions (Gadet, Pêcheux, Haroche, Authier-Revuz) constituant le volet IV « Discours et linguistique » du colloque (Conein et al., 1981 : 115-154).

88 Et plus spécifiquement du groupe «Analyse linguistique de la séquence », dont D. Maldidier (1990 : 77-79) retrace l'activité pendant les trois années d'existence (1981-1983) de la structure de recherche ADELA [Analyse du Discours Et Lecture d'Archive (CNRS)].

89 Pêcheux 1983 in (1990 : 300), cf. aussi Maldidier (1990: 78).

90 Ces formulations opposées figurant respectivement dans les « Mises au point [...] » sur l'AD de Pêcheux et Fuchs (1975), cité in Maldidier (1990 : 34), et dans une ultime «note » programmatique $\mathrm{du}$ « Livre blanc pour la recherche en linguistique » - parue de façon posthume (Pêcheux 1984). 
Pour saisir ce que D. Maldidier (1990 : 87) évoque comme un « déblocage du côté du sujet », il s'agit, serait-ce à grands traits, de dégager comment la contestation du sujet " souverain » $(\mathrm{A})$, reconnue ( $c f$. ci-dessus), comme un élément permanent de l'AD, s'y effectue en passant de la conception initiale d'un « non-sujet » (B) à celle, qui lui est irréductible, d'un sujet divisé (C). Le balisage qui suit a, si schématique qu'il soit, une double visée : celle, spécifique à l’AD, d'en éclairer l'évolution, au plan du sujet, solidaire des plans évoqués précédemment ( $i$, ii), et, au-delà, de façon plus générale, celle de mettre en place les repères minimaux que requiert, au plan du sujet, un traitement de la RDA. Si, en effet, toute approche de l'énonciation met en jeu - implicitement ou non - une conception du sujet et de son rapport au langage, c'est spécifiquement que le traitement de sa couche métalangagière se trouve conditionné par son « placement » dans le triangle (A)-(B)-(C) que structure crucialement la question de la (non)représentabilité, pour le sujet, de lui-même et du sens de son dire ${ }^{91}$.

\section{Repères dans le champ du sujet}

\subsection{Sujet plein et non-sujet : un face à face de narcissismes inversés}

Qu'il soit sommairement désigné comme « autonome, plein, origine... » le sujet $(A)$ déploie, en variantes psychologiques, sociales, neuronales..., la capacité fondamentale de " se connaître lui-même en tant que pensant ${ }^{92}$. Ce sujet transparent à lui-même exprime sa pensée avec les mots qu'il choisit, c'est un énonciateur qui « sait ce qu'il dit » et, le sachant, peut en parler, c'est-à-dire le représenter ; non que des « ratés » ne puissent survenir dans sa maîtrise du processus, ni que le « tout » de son sens et de sa pensée lui soit présent à l'esprit en permanence, mais « l'inconscient » n'y a le statut que d'un défaut, d'une défaillance cognitive contingente $-d u$ «non-conscient qui pourrait devenir conscient ${ }^{93}$.

Cette conception (A) du sujet est la plus « naturelle » et familière qui soit, puisqu'elle rejoint le sentiment spontané - et nécessaire - que les sujets parlants

91 Cf. Authier-Revuz (1995/2012), chapitre 2.

92 P. Schepens (2002a : 10) emprunte cette formulation à J.-P. Bronckart (1999), à la réflexion épistémologique duquel il s'appuie, en introduction à des contributions diverses sur le thème «Textes, Discours, Sujet » : rappelant que « dans le fond deux conceptions du sujet continuent à occuper le champ des sciences humaines ", il souligne un des points de clivage radical entre une « auto-conscience » héritière du Cogito (A) et conception post-freudienne (notre C) de l'inconscient.

93 Selon la formulation de Plon (2003). 
ont de contrôler leur dire. C'est celle que, travaillée dans leur cadre propre, reconduisent de façon dominante au plan théorique les sujets intentionnels de la pragmatique, les «stratèges » des interactions communicationnelles ou les régleurs (« monitoring ») de la machinerie verbale de la psycho-linguistique. Si une telle conception ouvre de plain-pied sur la RDA (ses stratégies, ses mises en scène...), la catégorie d'un « impensé » du dire lui est étrangère.

Le sujet (B), lui, se dessine dans le courant du structuralisme philosophique européen des années post-1960, au cœur de son grand mouvement de « démystification » des évidences " naturelles » du sujet humain touchant sa pensée, ses conduites, sa parole... par la mise au jour des mécanismes - non-subjectifs - qui structurellement les déterminent. Cette explosion de pensée, riche, hardie, n'est nullement monolithique ${ }^{94}$ : en ce qui concerne l'AD, c'est spécifiquement, à côté du Foucault de l'Archéologie du Savoir (1969) et de l'Ordre du Discours (1971), dans le sillage de la théorie des idéologies d'Althusser que sa conception du sujet a d'abord pris forme.

Figure inversée du (A), le sujet s'y résorbe - « évidé » - en pure illusion, dans ce qui (et, avec lui, son dire et son sens), intégralement, en est la cause réelle : « formations discursives » de Foucault, ensemble de règles anonymes, historiques, toujours déterminées dans le temps et dans l'espace qui y définissent « les conditions d'exercice de la fonction énonciative »; mécanisme idéologique, chez Althusser, de «l'assujettissement », c'est-à-dire de « l'interpellation des individus en sujets ${ }^{95}$. Sous les espèces de la « mort du sujet », réduit à une « fonction » ( $c f$. « la fonction auteur » de Foucault), ou de «l'effet-sujet », la formule ambiguë de « l'illusion subjective » ne renvoie pas dans ce contexte à une part de méconnaissance inhérente au fonctionnement subjectif, mais bien au sujet comme leurre.

Transposée au plan du discours, cette détermination " totale » est ce que postule l'AD 1 des Vérités de La Palice (1975) :

\footnotetext{
94 L'espace « structuraliste », aux contours d'ailleurs problématiques - Lévi-Strauss, Foucault, Althusser, Bourdieu, Barthes, Lacan, Derrida... - se partage notamment sur la question de la langue et de l'inconscient post-freudien (cf. ci-dessous) ; cf. par exemple les parcours - aux objectifs et points de vue sensiblement différents - qu'en présentent Dosse (1991-1992) et Milner (2002).

95 Comme toutes les évidences, y compris celles qui font qu'un mot « désigne une chose » ou " possède une signification », donc y compris les évidences de la « transparence du langage », cette évidence que vous et moi sommes des sujets - et que ça ne fait pas de problème - est un effet idéologique, l'effet idéologique élémentaire. (Althusser 1970 :30)
} 
on peut bien dire que l'intradiscours en tant que « fil du discours » du sujet est strictement un effet de l'interdiscours sur lui-même, une « intériorité » entièrement déterminée comme telle « de l'extérieur ». (Pêcheux (1990: 232)

Le « sujet doublement forclos » de l'AD ${ }_{1}$ que décrit D. Maldidier (1990 : 83-84) - forclos sur les deux versants de la co-énonciation, ramenée à "l'effet-sujet » d'un côté, à une " prothèse de lecture » de l'autre - s'inscrit dans ces théories philosophiques de la non-subjectivité. Et la démarche descriptive initiale, dans sa focalisation exclusive sur le réel des processus discursifs, au détriment de « l'inconsistance » des formes « de surface » que revêt le « leurre » énonciatif - a fortiori celles de son redoublement méta-énonciatif... - est solidaire de cet ancrage.

Il faut noter que les références à la théorie psychanalytique qui accompagnent la démarche de l'AD à ce stade [1] y sont, en revanche, exogènes : si on peut considérer que l'intérêt qu'elles manifestent annonce le vrai questionnement, qui se produira dans la dernière période [3], elles reposent en [1] sur la réduction de l'inconscient à l'idéologie, passant, centralement, par une confusion entre « l'imaginaire » chez Althusser - à quoi se ramène, dans son leurre, l'effet-sujet - et chez Lacan où il est instance d'un sujet divisé (et non pas annulé). Le retour critique de Pêcheux sur cette assimilation conditionne le déplacement de l'AD vers le sujet $(\mathrm{C})^{96}$.

L'antagonisme - radical - des sujets A et B est celui de leur narcissisme inversé $^{97}$ : celui d'un sujet souverain pour le premier, celui d'une structure toute puissante pour le second, qui se rejoignent - sujet plein/sujet vide - dans l'évitement de la division fondatrice du sujet post-freudien.

En ce qui concerne la prise en compte des deux étages du dire, celui des formes énonciatives par lesquelles il se représente et celui de l'extériorité dis-

96 Déplacement dont un des moments importants est l'ouvrage de P. Henry (1977) Le mauvais outil - Langue, Sujet et Discours, inscrivant au cœur de l'AD la conception psychanalytique d'un sujet divisé effet du langage - et non de «l'effet-sujet » ou « non-sujet » : « La psychanalyse traite le sujet comme un effet. Plus précisément, le sujet dont elle fait sa matière première est effet du langage. C'est en fin de compte cette mise en place du sujet par rapport au langage qui met la psychanalyse en position de rompre avec l'idéologie de la transparence. En outre poser le sujet comme effet exclut de le tenir pour centre, source, unité d'une intériorité, etc. » (p. 21).

97 J'emprunte ici au retour critique de Pêcheux en 1983 [in (1990 : 316)] sur l'AD ${ }_{1}$ : évoquant le « soupçon tout à fait explicite sur le registre du psychologique (et sur les psychologies du «moi » de la " conscience », du " comportement » ou du « sujet épistémique ») » issu de la pensée structuraliste, il souligne que « en même temps, ce mouvement anti-narcissique (dont les effets politiques et culturels ne sont visiblement pas épuisés) basculait dans une nouvelle forme de narcissisme théorique. Disons un narcissisme de la structure. » 
cursive dans laquelle il se détermine, le face à face des sujets A et B, le premier récusant le fait de la détermination qui constitue l'objet exclusif du second, laisse dans une impasse...

Remarque 4 : Approches du discours opposées par leur conception du sujet. En dépit de sa formulation en simples termes de choix « entre [...] deux directions de travail en analyse de discours » - et non d'une opposition entre deux conceptions du sujet - c'est bien sur ce fond d'un face à face entre sujets $\mathrm{A} / \mathrm{B}$ que se profile l'alternative par laquelle Ducrot répond à la critique des bases théoriques de la présupposition menée par P. Henry (1977) : lorsqu'il oppose d'un côté une sémantique prenant pour objet central « les représentations du sens » d'un énoncé par son énonciateur ${ }^{98}$, et de l'autre une AD qui « tenant pour une illusion l'éventualité que le locuteur soit sujet », se " désintéresse » de ces représentations au profit " d'un sens véritable " approché " selon des méthodes qui ne relèvent plus du tout de la recherche linguistique traditionnelle » (p. 181, 202-203).

\subsection{Le sujet divisé comme sortie d'une impasse}

Le sujet (C) « divisé », post-freudien, conteste assurément la maîtrise du sujet $\mathrm{A}$, transparent à lui-même et « sachant ce qu'il dit », mais non pas, à l'instar de la position B, l'existence d'un sujet ${ }^{99}$, singulier, et la réalité spécifique d'une parole dont l'écoute, dans sa lettre, matérielle, est cela même qui fonde la pratique psychanalytique.

Le sujet posé par Freud est

habité par un savoir qui lui échappe - l'inconscient, et mû par un désir dont les objets lui sont inconnus [S. Aouillé et al., 2010 : 123].

Et c'est en tant que, dans la parole du sujet, se fait entendre, à l'insu de celui-ci, le langage - évoqué par Proust - de son désir

ce magnifique langage, si différent de celui que nous parlons d'habitude, et où l'émotion fait dévier ce que nous voulions dire et épanouir à la place une phrase tout autre, émergée d'un lac inconnu où vivent des expressions sans rapport avec la pensée, et qui par cela même la révèlent ${ }^{100}$.

98 Position qui se radicalisera $(1980,1984)$ dans la conception, strictement intralinguistique, du sens d'un énoncé comme représentation, ou description, de son énonciation, $c f$. Authier-Revuz (1995/2012: 72-76), voir ci-dessous 3.4.2, p. 416.

99 «Le sujet est barré mais non absent ; il est manquant et non exterminé » (Roudinesco, 1977 : 43).

100 À la recherche du temps perdu, tome III, p. 822. Passage auquel fait allusion le titre du beau livre de Jean-Yves Tadié (2012) Le Lac inconnu - entre Proust et Freud. 
que cette parole - mise en jeu dans la cure - peut faire accéder le sujet à quelque chose de cet insu qui le gouverne : ainsi Lacan énonce-t-il que

La situation du sujet au niveau de l'inconscient telle que Freud l'articule [...] c'est qu'il ne sait pas avec quoi il parle, on a besoin de lui révéler les éléments proprement signifiants de son discours $[\ldots]^{101}$.

\section{caractérisant}

l'inconscient, à partir de Freud, [comme] une chaîne de signifiants qui quelque part (sur une autre scène, écrit-il) se répète et insiste pour interférer dans les coupures que lui offre le discours effectif [...] [Lacan $1966: 799$ ].

Reformulée par Lacan, dans son "retour à Freud », la conception d'un sujet qui n'est sujet que d'être parlant, et « produit » par le langage, comme structurellement clivé par un inconscient, ne relève aucunement des théories de la "non-subjectivité » : un « effet-sujet »-comme un mirage est un effet d'optique - ne se confond pas avec un "sujet, effet du langage ${ }^{102}$. Cet écart est très explicitement marqué par Lacan dans la discussion qui suit la présentation par Foucault de sa « fonction auteur ${ }^{103}$ : face aux tenants « de l'homme » et du « sujet » (au sens A), il oppose - se démarquant aussi par là de Foucault - à la notion de «négation du sujet » (B) imputée au structuralisme, celle

de la dépendance du sujet, ce qui est extrêmement différent ; et tout particulièrement, au niveau du retour à Freud, de la dépendance du sujet par rapport à quelque chose de vraiment élémentaire, et que nous avons tenté d'isoler sous le terme de « signifiant ».

B. Ogilvie (1987 : 42-43) analyse de façon aigüe cet échange comme révélateur de l'effort de Lacan pour « défaire » le sujet libre sans éliminer pour autant «le sujet $»^{104}$ : démarche par laquelle, précisément, le sujet (C), divisé, séparé qu'il est

101 Séminaire 1958 Le Désir, séance du 19-11-1958, cité in Mannoni (1969: 34), cf. aussi J. Dor (1985 : 132) : « Le langage apparaît donc comme cette activité subjective par laquelle on dit tout autre chose que ce que l'on croit dire dans ce que l'on dit. Ce « tout autre chose " s'instituant fondamentalement comme l'inconscient qui échappe au sujet qui parle parce qu'il en est fondamentalement séparé. »

102 Plus précisément « effet de la prise du corps dans le langage » (S. Aouillé et al. (2010 : 117) ou « ce qui surgit du vivant sous l'action du langage » (Miller (1981).

103 Foucault (1969b), repris in (1994: 820).

104 Ce que M. Plon (1988 : 246), dans un compte rendu de l'ouvrage d'Ogilvie, reformule en : «Lacan ne nie pas, n'invalide pas la question du sujet, se démarquant en cela d'un Michel Foucault, il ne cherche pas à éliminer un sujet au profit d'un autre, il ne rejette pas [...] le registre de 
de la part inconsciente de lui-même, offre - subvertissant l'opposition A/B - une sortie de «l'impasse » évoquée ci-dessus...

$\mathrm{Ni}$ accidentelle ou pathologique, ni contingente ou " remédiable » dans une unité retrouvée, la division freudienne du sujet, tenant spécifiquement au fait de l'existence - et de la dynamique - du savoir inconscient, est posée comme structurelle, constitutive.

On sait que Freud évoque les deux « blessures narcissiques » infligées à l'homme par Copernic - la terre n'est plus au centre du monde - puis par Darwin - il n'est plus, descendant du singe, au centre de la création - pour inscrire à leur suite la découverte d'un inconscient qui le décentre de lui-même, sans prise sur ce qui le cause. La " résistance » à cette dépossession de maîtrise est foncière : si on peut en débusquer, de façon critique, l'opiniâtre constance, sous la variété des formes qu'elle revêt au plan théorique ${ }^{105}$, elle est, pour le sujet, de l'ordre de la nécessité : au caractère structurel de la « division du sujet » répond, non moins structurelle, ce que Freud désignait comme « la fonction de méconnaissance du moi $»^{106}$.

Que la complexité du sujet de l'inconscient soit posée en termes de topique freudienne ou des structures de sujet élaborées par Lacan, le Moi-Imaginaire ${ }^{107}$ en est :

- une instance, à laquelle donc il ne se ramène pas ;

- une instance de " méprise », occupée à restaurer, pour le sujet, dans l'illusion, le sentiment de sa maîtrise ;

- une instance vitale faisant « tenir » le sujet qui, faute d'elle, se « défait ».

l'illusion mais situe le point nodal, celui d'un sujet vrai, sujet de l'inconscient à l'aune duquel ces représentations incontournables que ce sujet se donne de lui-même sous la forme de ces constructions illusoires que sont le sujet de la métaphysique ou le sujet de la psychologie peuvent être évaluées, nommées. » (idt).

105 Cf. le parcours effectué par J. Dor (1988) dans le champ : « [des] stratégies contre-subversives d'assujettissement ou de dénaturation [qui] n'ont cessé de rivaliser à [1']endroit [de la psychanalyse] aussi bien par l'ingéniosité de leurs modes d'articulation que par la diversité de leurs implications propres, [d'où il ressort que] malgré cette diversité, il est cependant quelque chose qu'elles ont toutes partagé, qui explicitement, qui implicitement : l'annulation de la dimension princeps convoquée par la psychanalyse, soit la "division du sujet”. » (Introduction, p. 14).

106 Cf. par exemple Roudinesco (1977: 42) : « Le sujet est décentré de sa position de maîtrise [...] Il est « divisé », mais pour autant il ne disparaît pas, il parle et continue dans le fantasme sous la forme du Moi. [...] La découverte de l'inconscient permet de signifier cette division inaugurale en montrant que l'illusion du centre demeure et qu'elle est inhérente à la constitution du sujet humain».

107 Voir, par exemple, Vanier (1998 : chap. II « L’imaginaire »). 
Ses formes, dès lors, - dans le dire comme ailleurs - requièrent l'attention pour ce qu'elles sont, sans les prendre (sujet A), ou au contraire les écarter (sujet B), pour ce qu'elles ne sont pas : le sujet lui-même (A) ou l'inconsistance d'un pur leurre (B); c'est ce droit de cité de l'imaginaire que souligne P. Clavreul (1987 : 79 ) au plan du sujet ${ }^{108}$ :

[L'imaginaire...] n'est pas ce truc vague, cette superstructure insolite dont il faut se méfier comme de la folle du logis, ou qu'il faut opposer à la solidité du réel, ou à celle du symbolique. Non, il n'y a pas un "imaginaire-caca" dont il faut se défier ; c'est au contraire quelque chose de consistant, à quoi Lacan a donné un statut tout aussi solide qu'au réel et au symbolique [...]. Sans l'imaginaire, le nœud borroméen ne tient donc plus et ouvre la porte à la folie.

\section{et que rappelait Flahaut (1978 : 153-154) dans son approche de la parole :}

l'écran [...] que nous interposons entre le fonctionnement réel de la parole et la conscience que nous en prenons [...] ne [doit] pas être considéré seulement négativement, comme une pure illusion sans épaisseur nous voilant la réalité : l'opacité est elle-même une certaine réalité [...]. [...] Ce qu'il nous faut regarder en face, c'est que le voile (avec ses effets d'illusion), nous ne pourrions vivre sans lui. Il s'agit donc de prendre au sérieux le superficiel, l'écume de la quotidienneté, la zone de tout ce qui vient conjurer l'insupportable surgissement du réel [...], l'espace où sont produites et où circulent des médiations dont la texture mêle le symbolique à l'imaginaire [...].

\subsection{Jalons sur le trajet de l'AD}

Les réceptions des Vérités de La Palice, dès lors qu'elles émanent de vrais lecteurs de Lacan, se rejoignent, dans des tonalités opposées - polémique pour Houde-

108 Je n'entre pas ici dans la conception « topologique » - à laquelle réfère Clavreul - du « nœud borroméen », liant entre elles les trois instances par lesquelles Lacan, à partir de 1953 dans « Le symbolique, l'imaginaire et le réel », structure le sujet. Très schématiquement, l'imaginaire est " lié à l'expérience d'un clivage entre le moi et le je (le sujet) », il se définit comme « le lieu du moi par excellence avec ses phénomènes d'illusion, de captation et de leurre », en rapport avec le symbolique comme lieu du signifiant de la loi du langage (et de la «fonction paternelle») et le réel comme « un reste impossible à symboliser » (d'après l'article « Imaginaire » in Roudinesco et Plon. 1997), le sujet tenant au nouage de ces trois instances. Sur l'évolution de Lacan depuis la « dévalorisation » initiale de la dimension imaginaire - solidaire de « l'exaltation du symbolique » dans les années 50, et de la critique de l'Ego-psychologie - jusqu'à sa reprise en compte comme instance « irréductible », faute de quoi un sujet ne peut tenir, voir Julien (1990 : parties 2 et 5 notamment) 
bine (1976), positive pour Plon (1976), en s'y appuyant pour Flahaut (1978) - pour y pointer de façon critique, ce qui y relève - dans la ligne du recouvrement de l'inconscient par l'idéologie chez Althusser ${ }^{109}$-, d'une assimilation (un « ravalement » dit Houdebine) du sujet au moi ${ }^{110}$.

C’est, entre autres, une réponse-auto-critique que leur adresse Pêcheux dans ce texte de 1978, qui fait rupture, " il n'y a de cause que de ce qui cloche ${ }^{\mathbf{1 1 1}}$, lorsqu'il prend acte que « l'ordre de l'inconscient ne coïncide pas avec celui de l'idéologie », et que " prendre trop au sérieux l'illusion d'un moi-sujet-plein où rien ne cloche [ $c f$. ci-dessus la détermination pensée comme « entière »], voilà précisément quelque chose qui cloche dans les Vérités de La Palice. »

Le déplacement qui s’y marque - depuis la « forme sujet idéologique » vers « l'existence d'une division du sujet, inscrite dans le symbolique »-, mis au travail dans la prise en compte, descriptive, de l'hétérogène au principe du discours et des événements énonciatifs singuliers qui, ouvrant avec l'inconscient sur l'équivoque de la langue, altèrent la prévisibilité du fil du discours, apparaît comme un « acquis » irréversible pour les textes de l'AD [3], dans la forêt de " questions », théoriques et opératoires, sur lesquelles il débouche ${ }^{112}$.

Dans ce mouvement qui quitte le sujet B, évidé, - et la visée $d u$ sens comme entièrement déterminé par une matérialité discursive historique - pour le sujet divisé d'un inconscient, singulier, irréductible à des processus discursifs, la théorie-pratique de l'AD se « décomplète »... À s'ouvrir sur l'hétérogène discursif,

$109 C f$., par exemple : « Depuis Marx, nous savons que le sujet humain [...] n'est pas le "centre” de l'histoire [...]. Freud nous découvre à son tour que le sujet [...] humain est décentré, constitué par une structure qui elle aussi n'a de " centre " que dans la méconnaissance imaginaire du “ moi ”, c'est-à-dire dans les formations idéologiques où il se " reconnaît” " (Althusser 1976 : 33-34).

110 Insistant sur « la distinction entre la constitution du sujet et celle du moi (c'est-à-dire des identifications du sujet) » (Flahaut 1978 : 156) ; sur la forclusion de la dimension singulière du sujet, «l'inconscient (individuel) et le sujet (singulier) [étant passés] à la moulinette bien connue de l'Histoire comme procès-sans-sujet » (Houdebine 1976) ; ou le caractère, inconciliable avec la théorie freudienne, du « sujet matérialiste issu de ce procès de dés-identification » (Plon 1976 : 95).

111 Mais dont l'avancée est comme "retenue », inédite en France avant son insertion dans (1990 : 261-272) par D. Maldidier ; voir à ce sujet les réflexions de Plon (2003).

112 Dans le détail desquelles je n'entrerai pas - non plus que dans la diversité des modes sur lesquels la réflexion s'est poursuivie à partir d'elles (« trajets » de corpus de Maldidier et Guilhaumou, algorithmes de mise en rapport séquence/mémoire de Marandin, notamment), $c f$. Authier-Revuz (1995/2012 : 251-252), Mazière (2005 : 56-58 et 67-70), ou dans des développements récents (Cislaru et Sitri 2012) sur la nécessaire hétérogénéité des corpus de mémoire évoquée ci-dessus 2.1.2 (i). 
l'équivoque de la langue, les formes de l'énonciation, des points d'émergence du sujet... l'AD renonce à donner accès « clefs en main » au sens d'un énoncé, c'est-àdire à l'ambition totalisante de ses débuts. Mais demeure, pour cette AD reconfigurée en pratique interprétative de " parcours de lecture » d'un énoncé dans des espaces de mémoire, la visée de quelque chose de ce qui se joue dans le sens entre une chaîne et la mémoire où elle se produit/reçoit.

À l'issue de ce parcours - et des trois déplacements qui s’y effectuent vers une extériorité interne hétérogène (i), la reconnaissance du fil énonciatif (ii), un sujet divisé (iii) -, l'AD, dans sa configuration [3], permet, théoriquement et descriptivement, d'aborder l'articulation des deux plans d'hétérogénéité discursive : constitutive du «ça parle ailleurs, avant et indépendamment » et représentée dans les formes métadiscursives de la RDA. Y apparaissent déterminants les points suivants :

1. la prise en compte pour l'énoncé de la double matérialité langagière où il se produit, celle (a) de l'épaisseur historique de la discursivité, et celle (b) du réel de la langue comme système de distinctivité et comme corps d'équivoque ;

2. la conception d'un sujet divisé pour lequel (a) le caractère inaccessible, irreprésentable du sens de ce qu'il dit - et notamment de la « mémoire » discursive où celui-ci prend corps - n'empêche nullement (b) la consistance singulière du fait de son énonciation - et de son « étage » de représentation métalangagière.

Remarque 5 : $\mathrm{AD}_{3}$ et dialogisme irréductibles l'un à l'autre. La forte solidarité théorique entre ces divers points ne permet pas de circonscrire la différence entre $\mathrm{AD}_{3}$ et dialogisme aux seuls points d'opposition, évidemment saillants, (1b) et (2a), du réel de la langue et du sujet divisé. Elle incite à contenir le rapprochement des deux approches, sur la base de ce qu'elles partagent en effet - la pensée d'une altérité discursive se jouant à la fois dans une extériorité produite par l'histoire et dans l'ici et maintenant du dire en train de se faire - dans les limites, loin d'une identification, d'une prudente mise en résonance ; ou bien de reconnaître que l'intégration de l'une au cadre d'ensemble de l'autre, ne va pas sans déplacement sensible du dit cadre.

\subsection{Le « sujet ", un espace polysémique à risque : flottements, glissades, malentendus}

Le domaine de la subjectivité est riche en faits de polysémie/homonymie propices aux flottements ou aux malentendus entre les conceptions $\mathrm{A}$, B et $\mathrm{C}$ distinguées ci-dessus, lorsque des termes s'y trouvent partagés - aux deux sens du mot, c'està-dire communs à un cadre et à un autre et divisés par les sens différents qu'ils y reçoivent. 


\subsubsection{Entre sujets (B) et (C) : non-sujet et sujet divisé}

On a noté ci-dessus la confusion dans l'AD $\mathrm{D}_{1}$, sous le vocable d' « imaginaire » entre les deux conceptions irréductibles d'Althusser et de Lacan - confusion solidaire de formulations du premier, assimilant inconscient et idéologie.

Il en va de même de la formulation de « l'illusion subjective », ou de " l'illusion constitutive du sujet », selon que ce syntagme s'explicite comme

(1) le sujet (n')est (qu')illusion ; l'illusion est d'être un sujet, qui correspondent à l'effet-sujet (B) des premières étapes de l'AD ; ou

(2) le sujet a une part, comporte, requiert de l'illusion; il y a de l'illusion dans le sujet,

qui relève du sujet divisé $(\mathrm{C})$.

Ainsi, reconnaître « de l'illusion » dans le fonctionnement subjectif n'implique pas que le sujet y disparaisse en «non-sujet » (B). La conception (C) est une théorie aussi subjective que la conception (A), en rapport de contestation avec celle-ci par la prise en compte de la division du sujet par l'inconscient.

Dans de nombreuses formulations - parfois " rituelles » comme celle du « retour du sujet » des années 80 - est en jeu le rabattement (ou non...) de la configuration triangulaire A-B-C sur l'opposition binaire A-B du sujet au non-sujet - en annexant à celui-ci, implicitement et indirectement, le sujet (C) divisé $\mathrm{e}^{113}$.

On peut certes penser que la caractérisation classique de l'AD comme " théorie non-subjective » ou " approche désubjectivisée du langage », adéquate, de fait, à la visée initiale de l’AD, relève seulement de l'amputation de la dernière période [3] de sa trajectoire. Mais une telle échappatoire n'est plus envisageable lorsque, par exemple, c'est l'approche que je propose de la couche méta-énonciative du dire - en tant qu'elle éclaire le rapport des sujets au langage et à ses non-coïncidences foncières ${ }^{114}$ - qui, pourtant explicitement associée à ses appuis freudo-lacaniens, se trouve définie comme un « travail de construc-

113 Évoquant « le grand retour du refoulé, le sujet » qui dans les années 80 a suivi le moment « anti-subjectif » structural - dont ne relève pas le sujet freudien revisité par Lacan - F. Dosse (1991 : 455 sq.) cite et commente ainsi des propos de F. Wahl : " "Tous ceux qui disent : Le sujet ? Le sujet ? comme de Gaulle disait : L'Europe ! L'Europe ! en se moquant de Lecanuet, me paraissent dérisoires parce que c'est un propos totalement impensé.” François Wahl vise ici tous ceux qui s'appuiraient dans leur retour au sujet sur la négation de sa division fondatrice, de sa structure clivée, au profit d'un sujet plein, d'une conception nouménale du sujet [...]. »

114 Authier-Revuz (1995/2012), notamment. 
tion d'une théorisation non subjective de l'énonciation ${ }^{115} »$ : cette formule dont le sens implique que « sujet » y signifie « sujet (A) », illustre exemplairement, dans un texte témoignant par ailleurs d'un souci aigu de précision, le caractère reçu, « établi » d'une telle assimilation ${ }^{116}$ (sujet = sujet (A)) dont on pourrait multiplier les exemples ${ }^{117}$...

Des flottements sur « imaginaire » ou « illusion subjective » on passe avec ces « théories non subjectives » recouvrant indistinctement les conceptions B et C, à une confusion dont le caractère usuel témoigne d'un contournement, d'autant plus efficacement consensuel qu'il est non-dit, de la division fondatrice du sujet : dans le face à face du sujet (A) au non-sujet (B) - aisé à mettre en défaut - le sujet divisé $(\mathrm{C})$, réduit à ce dernier $(\mathrm{B})$, n’a tout simplement pas droit de cité $^{118}$.

115 Bres et Rosier (2007 : 248), ou encore : « Dans sa [J. Authier] construction d'une théorie de l'énonciation non subjective et dans sa description des formes de non-coïncidences du dire [...] » (ibid. : 249).

116 À l'emprise de laquelle je n'ai pas échappé lorsque (Authier, 1984 : 89) j’oppose, sur un mode binaire, les conceptions (A), allant dans le sens des évidences des sujets parlants, à des théories non-subjectives du sujet et de la parole, allant contre ces mêmes évidences, renvoyant implicitement autant à ce qui relève de $\mathrm{B}$ (Foucault...) que de $\mathrm{C}$ (Freud, Lacan...).

117 Dans de nombreuses thèses de doctorat des trente dernières années, c'est comme sur le mode d'un passage obligé que figure - précédant les analyses (souvent pertinentes) de faits de discours (presse, entretiens...) qui constituent leur objet - le survol en quelques pages d'une histoire de l'analyse de discours en termes de passage du sujet " perdu », " complètement idéologique » (notre B), au sujet, heureusement " retrouvé », de la pragmatique, des interactions sociales, de la psychologie cognitive, etc. (relevant de A). Au delà de la simplification (faisant bon marché des questionnements internes à l'AD, de son évolution et des travaux résolument énonciatifs qui s'y rattachent, $c f$. ci-dessus 2.1.2. ii) p. 404 c'est, à ramener la question du sujet au remplacement (diachronique) du sujet B par le sujet A, le fait même de l'opposition («synchronique »), touchant la division du sujet, entre des conceptions du sujet qui est passé à la trappe.

118 On notera que le passage, chez Foucault, de la conception de l'individu « assujetti » (B), à celle des « sujets » et " modes de subjectivation » envisagés dans ses derniers séminaires (par exemple Le courage de la vérité, 2009) dans la visée de « la production de soi-même », n'implique pas une évolution de (B) vers le « sujet divisé » de (C), car ce travail et ses effets de subjectivité sont à ranger du côté du moi (cf. Aouillé et al. 2010 : 117). Je renvoie, repris dans Grignon (2017) aux pages éclairantes de Grignon (2008) « Avec quoi analyse-t-on ? », où questionnant dans la pratique psychanalytique elle-même « cette pente naturelle de chacun » à analyser, écouter, intervenir à partir de « ce moi auquel nous tenons tant » et non « en tant que sujet », il fait ce constat critique : «[...] le problème, c'est que nous avons des théories du sujet tellement misérables que quand nous pensons théorie du sujet, nous pensons théorie du moi ». (p. 84-85) 


\subsubsection{Entre sujets (A) et (C) - La « division » du sujet : démultiplication ou décentrement}

Le vocabulaire de la division et, solidairement, celui de " l'altérité » », densément convoqué dans le champ linguistico-discursif de l'énonciation, concentre les risques d'équivocité entre sens dans le cadre (A) et dans le cadre (C) post-freudien ${ }^{119}$.

Qu'il soit dit « divisé », " clivé », le sujet post-freudien, schématiquement évoqué ci-dessus, n'est pas un sujet qui se divise - se dédouble, se démultiplie -, il advient comme divisé, c'est-à-dire " séparé », « coupé » de la part inconsciente de lui-même, dont l'accès lui est « barré ». Parlant (et agissant) sans le savoir depuis un autre lieu, une « Autre Scène » qui lui est irreprésentable, c'est un sujet « où manque le fait d'une subjectivité psychologique » (Roudinesco $1977: 48$ ), c'est-à-dire auquel " centre » et " unité » font défaut. A l'aune de l' " hétéronomie radicale dont la découverte de Freud a montré dans l'homme la béance », " excentricité radicale de soi à lui-même à quoi l'homme est affronté » (Lacan 1966 : 524), la division du sujet (C) apparaît - loin des effets de dédoublement de soi, en rapport avec des autres, différents (de soi) - comme celle d'une altérité/ hétérogénéité constitutive où, pour le sujet, disparaît l'unité et le centre (hors le lieu « réparateur » de l'imaginaire).

Largement présentes dans la diversité des approches pragmatico-communicationnelles de l'énonciation, les notions de « division » (du sujet), d' « altérité », ou d' " hétérogénéité », caractérisées comme « internes » ou " constitutives », y relèvent d'une perspective tout à fait différente : celle d'une pluralité de voix, points de vue, images, rôles, personnages... dans laquelle « se distribue » le sujet parlant et/ou l'énonciation.

Il en est ainsi, par exemple, dans la théorie des sujets « de langage » de P. Charaudeau où la « mise en scène de la signification $~^{120}$, posée au principe de l'acte de langage, articule la double dimension d'altérité du dédoublement de l'un (1) en interférence (calculs, stratégies...) avec l'autre (2) :

[...] le sujet parlant est un être complexe, divisé parce qu'il a maille à partir avec les images qu'il se construit de l'autre comme interlocuteur et avec ce que peut être l'enjeu de l'acte langagier Charaudeau (1989: 9-10).

C'est aussi, bien entendu, le cas dans la conception polyphonique de l'énonciation développée à la suite de Ducrot dans laquelle la dimension de l'altérité est

119 Authier-Revuz (1995/2012 : 79-83 ; 88-92).

120 Charaudeau (1984), (1989). 
posée comme centrale : relevant du souci de « donner à l'altérité [...] une valeur constitutive », « [fondant] le sens sur l'altérité », la théorie de la polyphonie inscrit dans l'approche de l'énonciation

une altérité « interne » - en posant que le sens d'un énoncé décrit l'énonciation comme une sorte de dialogue cristallisé, où plusieurs voix s'entrechoquent (Ducrot 1984 : 9).

Cette altérité s'inscrit dans une conception de l'énonciation et du sens posée, doublement, comme représentation : passant du plan (1) de la définition du sens d'un énoncé comme « la représentation qu'il donne de son énonciation » (au sens de « ce qui est dit par l'énoncé à propos de son dire »), à celui (2) de cette « description du dire », conçue « comme une représentation théâtrale », dans laquelle un « locuteur responsable de l'énoncé donne existence au moyen de celui-ci à des énonciateurs dont il organise les points de vue et les attitudes » (Ducrot 1984 : 8-9).

Remarque 6 : Sur le sujet « polyphonique ». Il est hors de mon propos - et de ma portée - de parcourir, du point de vue de la question du « sujet », l'immense champ des études qui s'inscrivent dans - ou dans le sillage de - l'approche polyphonique de Ducrot ${ }^{121}$. C'est d'une façon schématique que j'indiquerai comment la prise en compte centrale d'une dimension de non-un dans le sens d'un dire où jouent des " voix » diverses, dans la polyphonie de Ducrot d'une part, dans le dialogisme bakhtinien, l'AD $\mathrm{D}_{3}$, et l'articulation « hétérogénéité représentée/constitutive » d'autre part, s’y effectue à des plans et selon des modes séparant irréductiblement la première des secondes.

Touchant la question du sujet parlant, le clivage se localise à deux niveaux : celui (a) de son exclusion dans la visée « intralinguistique » de la polyphonie, et celui (b) de la conception sous laquelle il fait cependant retour dans les descriptions.

121 Du cadre strictement pragmatique de Mœschler et Reboul (1994) aux élaborations syncrétiques de Rabatel (2008) de l'« homo narrans » (cf. p. 12-20), par exemple. Dans DAD (2002), Nølke donne une idée de ce que serait l'ampleur de la tâche lorsque, à l'article « Polyphonie », il souligne l'étendue et la diversité de la postérité de la théorie de Ducrot, « adoptée et adaptée par de nombreux chercheurs en linguistique et en analyse de discours ", notant que, dans le recours à cette notion, « les différentes acceptions divergent sur des points essentiels », observation réitérée à l'orée de leur travail par Nølke et al. (2004: 13) : « il y a presque autant de conceptions de cette notion que d'auteurs qui s'en servent ». (Je ne parle évidemment pas ici de l'emploi maximalement extensif du terme de « polyphonie », recensé dans le « Dictionnaire des Sciences du langage » de Neveu (2004), où la notion est envisagée, au delà de son introduction par Bakhtine, comme « par la suite approfondie en linguistique énonciative et en pragmatique (entre autres en France par Oswald Ducrot, Jean Claude Anscombre, Antoine Culioli, Alain Berrendonner, Jacqueline Authier-Revuz) [...] »). 
(a) La visée du projet polyphonique selon la formulation rituelle de « fin, remise en cause, en question... » du " postulat, dogme, mythe,... » de " l'unicité du sujet parlant ${ }^{122}$ est inscrite - ou enclose ? - dans ce que Rabatel (2005) appelle joliment une « scénographie interne à l'énoncé » : les entités plurielles mises en jeu dans la " représentation » énonciative - locuteur-metteur en scène et énonciateurs-personnages - reçoivent explicitement, chez Ducrot ${ }^{123}$, le statut d'êtres théoriques, posés sans rapport avec le sujet (réel, effectif, empirique) d'un acte de parole ${ }^{124}$. Posant que

Ducrot établit ainsi une frontière infranchissable entre le sujet parlant, c'est-à-dire l'individu dans le monde qui prononce l'énoncé, et le locuteur et les énonciateurs qui restent des êtres théoriques et qui ne s'incarnent pas.

Mœschler et al. (1994) en concluent, logiquement, que, dans son principe, « la théorie de Ducrot [...] ne traite en fait pas de la subjectivité ${ }^{125}$.

Si on prend acte de cette clôture « intralinguistique » - hors sujet, hors histoire, hors discours - postulée par la théorie polyphonique, celle-ci se place, avec « l'altérité » qu'elle pose centralement, en dehors du « triangle » des conceptions A-B-C du sujet, à l'écart de leurs choix opposés et « soustraite » par là, en droit, aux risques de glissements ou de confusions entre eux. C'est donc déjà à ce plan que, derrière leur « air de famille », l'altérité polyphonique d'une part, et ce que recouvre l'hétérogénéité constitutive du dire (où entrent dialogisme bakhtinien et $\mathrm{AD}$ [3]) se séparent : puisque la première se donne - représentation qu'un énoncé produit en lui-même de son énonciation - comme une image détachée du plan subjectif et historique de la production du dire, là où c'est justement à ce plan, de la parole d'un sujet dans l'histoire, qu'est reconnue la seconde, de l'ordre d'une extériorité interne au dire, à laquelle celui-ci ne peut pas se soustraire.

Aussi, au-delà de la reconnaissance, commune au dialogisme et à la polyphonie, d'une pluralité de voix jouant dans un énoncé, ne va-t-il pas sans forçage - ou flottement - de les ranger tous deux sous une même bannière de " mise en cause de l'unité du sujet parlant ${ }^{126}$, dans la mesure où la perspective polyphonique - intralinguistique et dans son principe a-subjective dans laquelle ce programme prend sens, est étrangère au dialogisme. C'est le cas, par exemple,

122 Cf. par exemple : Ducrot (1988 : 171, 172) ; Mœschler et al. $(1994$ : 324, 326).

123 Contrairement, par exemple, au modèle socio-communicatif de Charaudeau évoqué ci-dessus.

$124 C f$. « Je ne dis pas que l'énonciation c'est l'acte de quelqu'un qui produit un énoncé : [...] je ne veux pas prendre position [...]. » (Ducrot (1984 : 179), ou « faire abstraction du producteur empirique (position qui est aussi la mienne) » (ibid. : 172).

125 p. 326, 340, ou Nølke in (DAD 2002 : 445) : « Le rapport à l'être parlant réel n’intéresse pas O. Ducrot".

$126 \mathrm{Au}$ regard de l'exclusion explicite, hors du champ de la polyphonie, du « sujet-parlant » empirique, réel..., cette formulation peut être source de perplexité : s'agit-il d'un raccourci pour « sujet parlant représenté » comme non-unique, ou « image du sujet parlant comme nonunique », ou bien a-t-on à faire, dès cette formule de base, à l'oscillation, largement observable dans les développements de la polyphonie en analyse de discours, de part et d'autre de la « frontière infranchissable » séparant les « êtres théoriques » et les êtres « parleurs » ? 
dans Mœschler et Reboul (1994 : 323-324) à la démarche desquels renvoie Rabatel (2006 : 60), explicitant par un donc l'implication qui est l'objet de ma critique :

[les auteurs] font référence, en amont de Ducrot, à l'approche du dialogisme bakhtinien puisque les deux théoriciens ont insisté [a] « sur la multiplicité des points de vue exprimés dans un énoncé unique » (1994, p. 323), et donc [b] sur leur commun objectif de remettre en cause l'unicité du sujet parlant. (les [a] et [b] sont ajoutés par moi)

L'aventure dialogique, foncièrement subjective ${ }^{127}$, en quoi pour Bakhtine, on l'a vu, consiste pour la parole d'un sujet - « réel » - la traversée obligée du milieu touffu du déjà-dit, dans laquelle elle prend corps, ne se ramène pas à (pas plus que l'énoncé qui en résulte ne se prête à être représenté comme) une « régie théâtrale » maîtrisée de points de vue polyphoniques en présence.

(b) À supposer, maintenant, que l'« asepsie » intralinguistique soit « tenable », c'est-à-dire qu'il soit possible, dans le champ de l'énonciation et du sens, de s'affranchir du retour dans la description des extérieurs forclos du sujet et de l'histoire déposée dans les mots ${ }^{128}$, il est clair que dans la large mise en œuvre dans le champ énonciativo-discursif contemporain des catégories de la polyphonie, la " frontière infranchissable » - entre le sujet parlant producteur effectif de l'énoncé que la théorie choisit d'ignorer et les personnages de l'énonciation, locuteur et énonciateurs qu'elle institue - tend à s'estomper, ou disparaît, et, dans cette (re)mise en communication des deux plans, la " scénographie interne à l'énoncé " à se changer en - à se lester d'une conception de la subjectivité.

À franchir cette frontière, le « locuteur », de " personnage responsable de l'énoncé » qu'il était dans la représentation donnée par celui-ci, devient - « incarnation » favorisée par l'usage courant du terme - personne parlante, organisatrice, responsable du sens de ce qu'elle dit ${ }^{129}$. L'isomorphie entre la scénographie réflexive et la conception " naturelle » - illusoire et nécessaire - selon laquelle un sujet humain se représente en maître intentionnel de son dire, débouche - dispensée, par cette « naturalité » même, de s'expliciter comme théorie et de se justifier - dans une mise en œuvre du sujet (A), dont la polyphonie « subjectivisée » apparaît simplement comme

127 Est très présente chez Bakhtine, la dimension subjective - sur laquelle on revient dans les chapitres suivants - de l'expérience dialogique que nous faisons dans notre parole, doublement « altérée » de s'adresser à un autre à travers l'épaisseur historique du déjà-dit des autres discours : $c f$., entre mille, l'évocation de l'« attitude de dialogue [...] possible à l'égard [...] même d'un mot séparé s'il n'est pas considéré comme un mot impersonnel de la langue mais comme signe d'une position de sens appartenant à quelqu'un d'autre, comme représentant de l'énoncé d'un autre, c'est-à-dire si nous percevons en lui la voix d'un autre. » (Bakhtine 1929/1963 trad. fr. 1970 : 214, cité in Bres et Rosier 2007: 247).

128 Ce que je ne crois pas mais que je ne discuterai pas ici ( $c f$. Authier-Revuz 1995/2012 : 72-77). Voir, par exemple, ci-dessous (chap. 11.2.3, ex. (27), p. 477 : « étoiles nouvelles »), comment l'inévitable extérieur du déjà-dit vient ruiner le « calcul » intra-linguistique - polyphonique - du sens d'un guillemet.

129 Cf., par exemple, Vion (1998 : 199) évoquant cette pluralité de voix mise en jeu dans le dire comme « permettant au sujet de jouer à cache-cache avec des opinions, de les camper, de disparaître, de jouer une position en mineur ou en contrepoint, puis de se réapproprier, plus ou moins violemment une place énonciative dominante. » 
un variante sophistiquée dans laquelle, à passer de « soliste » à « chef de chœur » distribuant des voix conformément à ses intentions et sa stratégie, le sujet centré ne perd rien de sa souveraineté $\mathrm{e}^{130}$.

Retrouvant ainsi le terrain de la subjectivité à l'écart duquel la visée intralinguistique prétendait la tenir, la polyphonie retrouve les clivages qui le traversent et - catalysés par la problématique de l' « altérité »- les glissements et confusions dont il est le lieu. La confusion principale se joue entre sujets (A) et (C), régulièrement dans le sens d'un recouvrement de l'inconfort narcissique du second par l'« évidence » sécurisante du premier : l'unité subvertie par une division hétérogénéisante de (C) est « rapatriée » dans l'unicité déployée en pluralité diversifiante de (A).

Du recouvrement de (C) par (A), l'échange intertextuel suivant ${ }^{131}$ présente, dans son malentendu, une forme exemplairement explicite: A. Grésillon (1979 : 11) opposant, à propos du traitement de la présupposition par Ducrot, la conception du sujet (A) «plein », intentionnel, origine et cause de ses pensées et stratégies langagière qui y est mise en œuvre, à celle d'un sujet (C), décentré et divisé d'être " pris dans un ensemble de déterminations idéologiques et psychanalytiques », conclut, à mon avis pertinemment, que

la théorie des actes de langage reste prisonnière de la problématique subjectiviste centrée sur l'individu et [...] refuse de mettre l'unité du sujet en question.

Une dizaine d'années plus tard, C. Kerbrat (1991 : 16) caractérise cette critique comme désormais obsolète, la pragmatique s'étant largement ouverte sur la pluralité des voix...

Si en 1979, Grésillon pouvait encore et à juste titre reprocher aux pragmaticiens une conception archaïque et monolithique du sujet parlant [...] les temps ont depuis bien changé : la polyphonie est désormais partout [...] on la traque dans les énoncés les plus innocemment

130 Outre à (Maldidier et al. 1985: 46) jugeant, à propos du « raffinement apporté autour du problème du sujet » par la démultiplication polyphonique, qu'on « peut douter, [...] au-delà des subtilités qu'il permet dans l'analyse, qu'il marque une rupture dans la problématique antérieure d'un sujet "responsable d'un acte de parole" ", je renvoie aux analyses formulées antérieurement dans Authier-Revuz (1995/2012 : 79-95) et (1998 : 70), notamment : « L'autre, l'altérité, la division dont il est question ici [...] s'inscrit dans l'espace propre à la psychologie sociale qui est celui d'un jeu interactif, spéculaire, des intentionnalités. Le sujet metteur en scène, si nombreux que soient les rôles dans lesquels il se démultiplie, demeure fondamentalement UN, en tant que centré et "maître en sa propre demeure" pour parler comme Freud. ». Cf. encore Rabatel (2005: 11) motivant ses propositions de déplacement dans l'appréhension d'instances plurielles (locuteurs/ énonciateurs) par la prise en compte (référant notamment aux textes ci-dessus) du « risque de dérives idéalistes certaines » de l'approche polyphonique.

131 Analysé plus précisément dans Authier-Revuz (1995/2012 : 90-91). 
monodiques en apparence, et ce qui menace le sujet c'est aujourd'hui bien plutôt une atomisation excessive [...].

Ce long détour à travers un champ dont les clivages profonds sont si aisément recouverts par la polysémie qui y règne, tient à ce que, au rebours du caractère dominant du sujet (A) - et des rabattements qu'il entraîne... - l'ancrage dans la conception (C) d'un sujet divisé conditionne, pour la RDA, l'approche, qui fait l'objet de ce qui suit, de sa fonction dans l'économie du dire.

Le statut que l'on peut reconnaître à la RDA dans un discours, c'est-à-dire à l'hétérogénéité qu'il représente en lui même par des formes métadiscursives, dépend, en effet, foncièrement des choix effectués aux deux plans évoqués dans ce chapitre : celui de la reconnaissance - ou non - d'une hétérogénéité constitutive du dire, que celui-ci n'est pas à même de représenter ; et au plan de la complexité d'un sujet non-un, la reconnaissance - ou non - en deçà de la pluralité à laquelle se prête le sujet (A) centré, d'une division séparant le sujet (C) d'une part de lui-même et du sens de ce qu'il dit, requérant la restauration, dans l'imaginaire, d'une centration de type (A). 